University of Louisville

ThinkIR: The University of Louisville's Institutional Repository

Electronic Theses and Dissertations

$8-2020$

\title{
Convolution inequalities and applications to partial differential equations.
}

Matthew Reynolds

University of Louisville

Follow this and additional works at: https://ir.library.louisville.edu/etd

Part of the Partial Differential Equations Commons

\section{Recommended Citation}

Reynolds, Matthew, "Convolution inequalities and applications to partial differential equations." (2020). Electronic Theses and Dissertations. Paper 3512.

https://doi.org/10.18297/etd/3512

This Doctoral Dissertation is brought to you for free and open access by ThinkIR: The University of Louisville's Institutional Repository. It has been accepted for inclusion in Electronic Theses and Dissertations by an authorized administrator of ThinkIR: The University of Louisville's Institutional Repository. This title appears here courtesy of the author, who has retained all other copyrights. For more information, please contact thinkir@louisville.edu. 


\title{
CONVOLUTION INEQUALITIES AND APPLICATIONS TO PARTIAL DIFFERENTIAL EQUATIONS
}

\author{
by \\ Matthew Reynolds \\ B.S. University of Louisville, 2014 \\ M.A. University of Louisville, 2016 \\ July 28,2020

\begin{abstract}
A Dissertation
Submitted to the Faculty of the

College of Arts and Sciences of the University of Louisville

in Partial Fulfillment of the Requirements

for the Degree of
\end{abstract} \\ Doctor of Philosophy \\ in \\ Applied and Industrial Mathematics \\ Department of Mathematics \\ University of Louisville \\ Louisville, KY
}

August 2020 



\title{
CONVOLUTION INEQUALITIES AND APPLICATIONS TO PARTIAL DIFFERENTIAL EQUATIONS
}

\author{
Submitted by \\ Matthew Reynolds
}

A dissertation approved on

July 28, 2020

by the Following Dissertation Committee

Dr. David Swanson,

Dissertation Director

Dr. David Brown

Dr. Changbing $\mathrm{Hu}$

Dr. Lee Larson 
For Mom, Dad, Zelda, Samus, and Lara 


\section{ACKNOWLEDGMENTS}

I'd like to thank the following for their invaluable contributions.

- Thanks to my Mom and Dad for believing in me even when I did not.

- Thanks to my friend Israel Ricardo Curbelo for introducing me to the joy of waifus, without whom I surely would have gone insane on this lonesome mathematical journey.

- Thanks to Qi, for being there for me at every step.

- Thanks to my friend Trevor Leach for his last minute help with typesetting issues.

- Thanks to my dissertation committee for their invaluable insight, criticism, and praise each of which helped this dissertation reach its full potential.

- Thanks to my advisor, David Swanson for his continued mentorship.

- Finally, thanks to Samus Aran for her symbolic reminders of the value of strength, courage, willpower, and perseverence in the face of pain and doubt. 


\begin{abstract}
CONVOLUTION INEQUALITIES AND APPLICATIONS TO PARTIAL DIFFERENTIAL EQUATIONS
\end{abstract}

Matthew Reynolds

July 28,2020

In this dissertation we develop methods for obtaining the existence of mild solutions to certain partial differential equations with initial data in weighted $L^{p}$ spaces and apply them to some examples as well as improve the solutions to some known PDEs studied extensively in the literature. We begin by obtaining a version of a Stein-Weiss integral inequality which we will use to obtain general convolution inequalities in weighted $L^{p}$ spaces using the techniques of interpolation. We will then use these convolution inequalities to make estimates on PDEs that will help us obtain mild solutions as fixed points of certain contraction mappings. Then Lorentz spaces will be introduced and interpolation will be used again to obtain convolution inequalities in weighted Lorentz spaces. Finally, the possibility of investigating PDEs with initial data in weighted Lorentz spaces will be discussed. 
TABLE OF CONTENTS

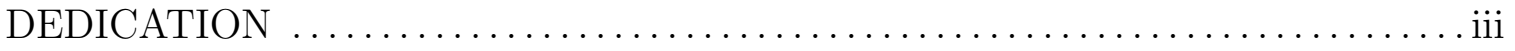

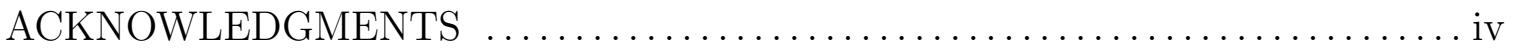

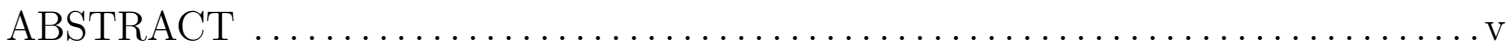

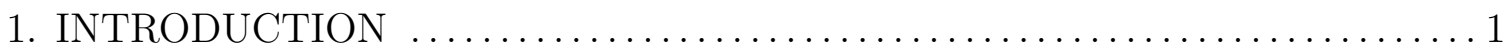

2. A STEIN-WEISS INTEGRAL INEQUALITY WITH NEW WEIGHTS $\ldots \ldots .1$

3. CONVOLUTION INEQUALITIES IN WEIGHTED $L_{p}$ SPACES $\ldots \ldots \ldots \ldots . . .19$

4. FORMULATION OF A MILD SOLUTION $\ldots \ldots \ldots \ldots \ldots \ldots \ldots \ldots \ldots \ldots$

5. FINDING A MILD SOLUTION TO A SIMPLE PDE $\ldots \ldots \ldots \ldots \ldots \ldots \ldots . \ldots 37$

6. IMPROVED SOLUTIONS TO THE KURAMOTO-SIVASHINSKY

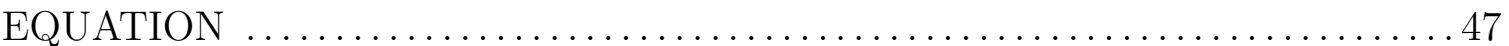

7. CONVOLUTION INEQUALITIES IN WEIGHTED LORENTZ SPACES ...52

8. OPPORTUNITIES FOR FURTHER RESEARCH $\ldots \ldots \ldots \ldots \ldots \ldots$

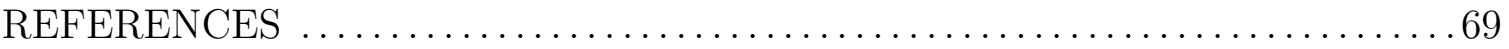

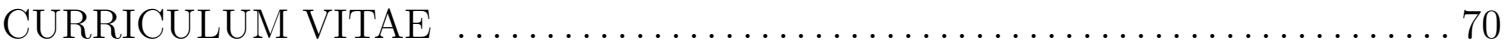




\section{CHAPTER 1}

\section{INTRODUCTION}

\subsection{PRELIMINARIES}

We begin with some notation and definitions. Throughout this dissertation we will frequently refer to Banach spaces. This means the usual normed vector space that is complete with respect to the metric induced by the norm. Particularly we will be interested in Banach Spaces in the form of weighted $L^{p}$ spaces. To this end we begin by defining the usual $L^{p}$ space with respect to an arbitrary measure.

Definition 1.1: ( $L^{p}$ spaces) Let $u: \mathbb{R}^{n} \rightarrow \mathbb{C}$ be measurable with respect to the measure $\nu$. Define a functional, called the $p$-norm, like so:

$$
\|u\|_{p}=\left(\int_{\mathbb{R}^{n}}|u|^{p} d \nu\right)^{\frac{1}{p}}
$$

Then the usual $L^{p}$ space with respect to the measure $\nu$ on $\mathbb{R}^{n}$ is defined as:

$$
L^{p}\left(\mathbb{R}^{n}\right)=\left\{u: \mathbb{R}^{n} \rightarrow \mathbb{C} \mid\|u\|_{p}<\infty\right\}
$$

There is an analogous more general definition of $L^{p}$ spaces for arbitrary measure spaces instead of just $\mathbb{R}^{n}$ but this dissertation will focus on $\mathbb{R}^{n}$. It's well known that $L^{p}$ spaces are Banach spaces with respect to the $p$-norm. When $L^{p}$ spaces are defined with respect to arbitrary measures, the weighted $L^{p}$ spaces are in fact a special case of the usual ones.

Definition 1.2: (Weighted $L^{p}$ spaces) A weighted $L^{p}$ space is just a usual $L^{p}$ 
space where the underlying measure $\nu$ is Radon-Nikodym differentiable with respect to Lebesgue measure.

When $w(x)$ is the Radon-Nikodym derivative of $\nu$ we will write $w(x) d x$ in place of $d \nu$. In this dissertation we will concern ourselves mostly with weights of the form $w(x)=(1+|x|)^{\alpha p}$ for reasons that become clear later. Therefore we will use two forms of notation for norms, namely $\|u\|_{w, p}$ when the underlying measure is $w(x) d x$ and $\|u\|_{\alpha, p}$ when $w(x)=(1+|x|)^{\alpha p}$. This pattern will be abolished in the chapter on Lorentz spaces in order to make how certain theorems are being applied more apparent. In that chapter the distinction will be made clear. Next we define the convolution of two functions $f$ and $g$.

Definition 1.3: (Convolution of two functions) The convolution of two measureable functions $f$ and $g$ is denoted by $f * g(x)$ and is given by:

$$
f * g(x)=\int_{\mathbb{R}^{n}} f(y) g(x-y) d y
$$

In our study of mild solutions of partial differential equations it will be useful to obtain bounds on norms of convolutions of the following form:

$$
\|f * g\|_{\theta ; a} \leq C\|f\|_{\gamma ; b}\|g\|_{\sigma ; c}
$$

where the constant $C$ is independent of functions $f$ and $g$. Inequalities of this form are the convolution inequalities to which we will frequently refer. However the convolution operator is only a bilinear operator and sometimes we will present theorems about multilinear operators. In those cases we will use:

$$
T: L_{p_{1}, u_{1}} \times \ldots \times L_{p_{l}, u_{l}}\left(\mathbb{R}^{n}\right) \rightarrow L_{p, u}\left(\mathbb{R}^{n}\right)
$$


to mean that $T$ is an $l$-linear operator from $L_{p_{1}, u_{1}} \times \ldots \times L_{p_{l}, u_{l}}\left(\mathbb{R}^{n}\right)$ into $L_{p, u}\left(\mathbb{R}^{n}\right)$. If the norm of the operator is needed it will be stated explicitly. 


\section{CHAPTER 2}

\section{A STEIN-WEISS INTEGRAL INEQUALITY WITH NEW WEIGHTS}

\subsection{A USEFUL INTEGRAL INEQUALITY}

Our development of useful convolution inequalities will require obtaining bounds on norms of operators of the form:

$$
T_{\lambda, \alpha, \beta}(f)=\int_{\mathbb{R}^{n}} \frac{f(y)}{w_{\beta}(x) w_{\lambda}(x-y) w_{\alpha}(y)} d y
$$

Building on the work of Hardy and Littlewood [1], Stein and Weiss [2] obtained such bounds when $w(x)=|x|^{\alpha}$. A simplified proof of this result was offered by Swanson [3]. In this chapter we will adapt the methods of Swanson to obtain a similar result of Stein and Weiss for weights of the form $w(x)=(1+|x|)^{\alpha}$. We will see these weights arise naturally in our study of partial differential equations. To this end, for $f \in L^{p}\left(\mathbb{R}^{n}\right)$, define:

$$
T f(x)=\int_{\mathbb{R}^{n}} \frac{f(y)}{(1+|x|)^{\alpha}(1+|x-y|)^{\lambda}(1+|y|)^{\beta}} d y
$$

The following theorem is the main theorem of this chapter.

Theorem 2.1 Suppose that $1<p \leq q<\infty$. Then there exists a constant $C$ independent of $f$ such that $\|T f\|_{q} \leq C\|f\|_{p}$ if and only if the following holds:
a) $\alpha+\lambda>\frac{n}{q}$
b) $\beta+\lambda>\frac{n}{p^{\prime}}$
c) $\alpha+\beta+\lambda \geq \frac{n}{p^{\prime}}+\frac{n}{q}$ 
d) $\alpha+\beta \geq 0$

e) $\alpha+\beta+\lambda=n$ and $p=q \Longrightarrow \lambda<n$

The proof of theorem 2.1 will involve writing the operator $T$ as a sum of three operators $T_{1}, T_{2}$, and $T_{3}$ that we estimate individually.

\subsection{PROOF OF INEQUALITY}

For nonnegative measureable $f: \mathbb{R}^{n} \rightarrow \mathbb{R}$ define:

$$
T_{1, \gamma, \beta} f(x)=(1+|x|)^{\gamma} \int_{|y| \leq|x|} \frac{f(y)}{(1+|y|)^{\beta}} d y
$$

Lemma 2.2: Suppose that $1<p \leq q<\infty$. Then there is a constant $C$ independent of $f$ such that $\left\|T_{1, \gamma, \beta} f\right\|_{q} \leq C\|f\|_{p}$ if and only if $\gamma-\beta \leq-n\left(\frac{1}{p^{\prime}}+\frac{1}{q}\right)$ and $\gamma<-\frac{n}{q}$.

First we prove sufficiency for the $p=q$ case. Suppose that $\gamma-\beta \leq-n$ and $\gamma<-\frac{n}{p}$.

$$
\begin{aligned}
\left\|T_{1, \gamma, \beta} f\right\|_{p} & =\left(\int_{\mathbb{R}^{n}}\left((1+|x|)^{\gamma} \int_{|y| \leq|x|} \frac{f(y)}{(1+|y|)^{\beta}} d y\right)^{p} d x\right)^{1 / p} \\
= & \left(\int_{\mathbb{R}^{n}}\left(\int_{|y| \leq|x|} \frac{(1+|x|)^{\gamma}}{(1+|y|)^{\beta}} f(y) d y\right)^{p} d x\right)^{1 / p}
\end{aligned}
$$

Now we switch to polar coordinates and let $y=r \omega$ with $|y|=r$ and then apply Minkowski's inequality:

$$
\begin{gathered}
\left\|T_{1, \gamma, \beta} f\right\|_{p}=\left(\int_{\mathbb{R}^{n}}\left(\int_{S^{n-1}}\left(\int_{0}^{|x|} \frac{(1+|x|)^{\gamma}}{(1+r)^{\beta}} f(r \omega) r^{n-1} d r\right) d \omega\right)^{p} d x\right)^{1 / p} \\
\leq \int_{S^{n-1}}\left(\int_{\mathbb{R}^{n}}\left(\int_{0}^{|x|} \frac{(1+|x|)^{\gamma}}{(1+r)^{\beta}} f(r \omega) r^{n-1} d r\right)^{p} d x\right)^{1 / p} d \omega
\end{gathered}
$$


Switching coordinates again using $x=s \theta,|x|=s$ and recognizing that $\left(\int_{S^{n-1}} d \theta\right)^{1 / p}$ is a constant yields:

$$
\begin{gathered}
\left\|T_{1, \gamma, \beta} f\right\|_{p}=\int_{S^{n-1}}\left(\int_{S^{n-1}} \int_{0}^{\infty}\left(\int_{0}^{s} \frac{(1+s)^{\gamma}}{(1+r)^{\beta}} f(r \omega) r^{n-1} d r\right)^{p} s^{n-1} d s d \theta\right)^{1 / p} d \omega \\
\leq C \int_{S^{n-1}}\left(\int_{0}^{\infty}\left(\int_{0}^{s} \frac{(1+s)^{\gamma}}{(1+r)^{\beta}} f(r \omega) r^{n-1}\left(s^{n-1}\right)^{1 / p} d r\right)^{p} d s\right)^{1 / p} d \omega
\end{gathered}
$$

Substituting $r=s t$ with $d r=s d t$, applying Minkowski's inequality, and finally Fubini's theorem gives:

$$
\begin{aligned}
\left\|T_{1, \gamma, \beta} f\right\|_{p} \leq C \int_{S^{n-1}}\left(\int_{0}^{\infty}\left(\int_{0}^{1} \frac{(1+s)^{\gamma}}{(1+s t)^{\beta}} f(s t \omega) s^{n} t^{n-1}\left(s^{n-1}\right)^{1 / p} d t\right)^{p} d s\right)^{1 / p} d \omega \\
\leq C \int_{S^{n-1}} \int_{0}^{1}\left(\int_{0}^{\infty}\left(\frac{(1+s)^{\gamma}}{(1+s t)^{\beta}} f(s t \omega) s^{n} t^{n-1}\right)^{p} s^{n-1} d s\right)^{1 / p} d t d \omega \\
\leq C \int_{0}^{1} \int_{S^{n-1}}\left(\int_{0}^{\infty}\left(\frac{(1+s)^{\gamma}}{(1+s t)^{\beta}} f(s t \omega) s^{n} t^{n-1}\right)^{p} s^{n-1} d s\right)^{1 / p} d \omega d t
\end{aligned}
$$

We consider the case the case $\gamma+n>0$. When $0<t<1$ we have:

$$
\frac{(1+s)^{\gamma}}{(1+s t)^{\beta}} s^{n} \leq \frac{(1+s)^{\gamma}}{(1+s t)^{\gamma+n}} s^{n} \leq\left(\frac{1+s}{1+s t}\right)^{\gamma+n} \leq t^{-\gamma-n}
$$

Applying Holder's inequality to the integral over $S^{n-1}$ and then applying this estimate gives:

$$
\begin{gathered}
\int_{S^{n-1}}\left(\int_{0}^{\infty}\left(\frac{(1+s)^{\gamma}}{(1+s t)^{\beta}} f(s t \omega) s^{n} t^{n-1}\right)^{p} s^{n-1} d s\right)^{1 / p} d \omega \\
\leq\left(\int_{S^{n-1}} \int_{0}^{\infty}\left(\frac{(1+s)^{\gamma}}{(1+s t)^{\beta}} f(s t \omega) s^{n} t^{n-1}\right)^{p} s^{n-1} d s d \omega\right)^{1 / p}\left(\int_{S^{n-1}} 1^{p^{\prime}} d w\right)^{1 / p^{\prime}}
\end{gathered}
$$




$$
\begin{gathered}
=C\left(\int_{S^{n-1}} \int_{0}^{\infty}\left(\frac{(1+s)^{\gamma}}{(1+s t)^{\beta}} f(s t \omega) s^{n} t^{n-1}\right)^{p} s^{n-1} d s d \omega\right)^{1 / p} \\
\leq C\left(\int_{S^{n-1}} \int_{0}^{\infty}\left(f(s t \omega) t^{-\gamma-1}\right)^{p} s^{n-1} d s d \omega\right)^{1 / p} \\
=C t^{-\gamma-1}\left(\int_{S^{n-1}} \int_{0}^{\infty} f(s t \omega)^{p} s^{n-1} d s d \omega\right)^{1 / p}
\end{gathered}
$$

Combining (2) with the substitution $s=\frac{1}{t} r$ with $d s=\frac{1}{t} d r$ gives:

$$
\begin{gathered}
\int_{S^{n-1}}\left(\int_{0}^{\infty}\left(\frac{(1+s)^{\gamma}}{(1+s t)^{\beta}} f(s t \omega) s^{n} t^{n-1}\right)^{p} s^{n-1} d s\right)^{1 / p} d \omega \\
\leq C t^{-\gamma-1}\left(\int_{S^{n-1}} \int_{0}^{\infty} f(r \omega)^{p} \frac{r^{n-1}}{t^{n-1}} * \frac{1}{t} d r d \omega\right)^{1 / p} \\
=C t^{-\gamma-1-n / p}\left(\int_{S^{n-1}} \int_{0}^{\infty} f(r \omega)^{p} r^{n-1} d r d \omega\right)^{1 / p} \\
=C t^{-\gamma-1-n / p}\|f\|_{p}
\end{gathered}
$$

The assumptions on $\gamma$ imply that $-\gamma-1-n / p>-1$. Combining this with (1) and (3) gives:

$$
\left\|T_{1, \gamma, \beta} f\right\|_{p} \leq C \int_{0}^{1} t^{-\gamma-1-n / p}\|f\|_{p} d t=C\|f\|_{p}
$$

Alternatively suppose that $\gamma+n \leq 0$. For $0<t<1$ we have:

$$
\frac{(1+s)^{\gamma}}{(1+s t)^{\beta}} s^{n} \leq \frac{(1+s)^{\gamma}}{(1+s t)^{\gamma+n}} s^{n} \leq \frac{(1+s)^{\gamma+n}}{(1+s)^{\gamma+n}}=1
$$

Similarly, we apply Holder's inequality, the above estimate, and again substitute $s=\frac{1}{t} r$ giving:

$$
\int_{S^{n-1}}\left(\int_{0}^{\infty}\left(\frac{(1+s)^{\gamma}}{(1+s t)^{\beta}} f(s t \omega) s^{n} t^{n-1}\right)^{p} s^{n-1} d s\right)^{1 / p} d \omega
$$




$$
\begin{gathered}
\leq C\left(\int_{S^{n-1}} \int_{0}^{\infty}\left(f(s t \omega) t^{n-1}\right)^{p} s^{n-1} d s d \omega\right)^{1 / p} \\
\leq C\left(\int_{S^{n-1}} \int_{0}^{\infty}\left(f(r \omega) t^{n-1}\right)^{p} \frac{r^{n-1}}{t^{n-1}} * \frac{1}{t} d r d \omega\right)^{1 / p} \\
=C t^{n-1-n / p}\|f\|_{p}
\end{gathered}
$$

Noting that $n-1-n / p>-1$ we obtain:

$$
\left\|T_{1, \gamma, \beta} f\right\|_{p} \leq C \int_{0}^{1} t^{n-1-n / p}\|f\|_{p} d t=C\|f\|_{p}
$$

Thus the conditions on $\gamma$ and $\beta$ are sufficient for the $p=q$ case. We now generalize and assume $1<p<q<\infty$ and that

$$
\gamma-\beta \leq-n\left(\frac{1}{p^{\prime}}+\frac{1}{q}\right), \quad \gamma<-\frac{n}{q}
$$

We have that:

$$
\begin{aligned}
\left(T_{1, \gamma, \beta} f(x)\right)^{q} & =(1+|x|)^{\gamma q}\left(\int_{|y| \leq|x|} \frac{f(y)}{(1+|y|)^{\beta}} d y\right)^{q-p}\left(\int_{|y| \leq|x|} \frac{f(y)}{(1+|y|)^{\beta}} d y\right)^{p} \\
\leq(1+|x|)^{\gamma q} & \left(\int_{|y| \leq|x|} \frac{f(y)}{(1+|y|)^{\gamma+n\left(1 / p^{\prime}+1 / q\right)}} d y\right)^{q-p}\left(\int_{|y| \leq|x|} \frac{f(y)}{(1+|y|)^{\beta}} d y\right)^{p} \\
= & (1+|x|)^{\gamma q+p n-p \gamma-p n\left(1 / p^{\prime}+1 / q\right)}\left(\int_{|y| \leq|x|} \frac{f(y)}{(1+|y|)^{\gamma+n\left(1 / p^{\prime}+1 / q\right)}} d y\right)^{q-p} \\
& \times\left((1+|x|)^{\gamma+n\left(1 / p^{\prime}+1 / q\right)-n} \int_{|y| \leq|x|} \frac{f(y)}{(1+|y|)^{\beta}} d y\right)^{p}
\end{aligned}
$$

Now, Holder's inequality and elementary calculus tells us that:

$$
\int_{|y| \leq|x|} \frac{f(y)}{(1+|y|)^{\gamma+n\left(1 / p^{\prime}+1 / q\right)}} d y
$$




$$
\begin{gathered}
\leq\|f\|_{p}\left(\int_{|y| \leq|x|} \frac{1}{(1+|y|)^{\left(\gamma+n\left(1 / p^{\prime}+1 / q\right)\right) p^{\prime}}} d y\right)^{1 / p^{\prime}} \\
\leq C\|f\|_{p}(1+|x|)^{n / p^{\prime}-\gamma-n\left(1 / p^{\prime}+1 / q\right)}
\end{gathered}
$$

Combining (4) and (5) and recognizing that

$$
\gamma q+p n-p \gamma-p n\left(1 / p^{\prime}+1 / q\right)+\left(n / p^{\prime}-\gamma-n\left(1 / p^{\prime}+1 / q\right)\right)(q-p)=0
$$

we have:

$$
\left(T_{1, \gamma, \beta} f(x)\right)^{q} \leq C\|f\|_{p}^{q-p}\left((1+|x|)^{\gamma+n\left(1 / p^{\prime}+1 / q\right)-n} \int_{|y| \leq|x|} \frac{f(y)}{(1+|y|)^{\beta}} d y\right)^{p}
$$

Since $\gamma+n\left(1 / p^{\prime}+1 / q\right)-n-\beta \leq-n$ and $\gamma+n\left(1 / p^{\prime}+1 / q\right)-n<-n / p$ we may combine (6) with the $p=q$ case to obtain:

$$
\begin{gathered}
\left\|T_{1, \gamma, \beta} f\right\|_{q} \leq C\left(\int_{\mathbb{R}^{n}}\|f\|_{p}^{q-p}\left((1+|x|)^{\gamma+n\left(1 / p^{\prime}+1 / q\right)-n} \int_{|y| \leq|x|} \frac{f(y)}{(1+|y|)^{\beta}} d y\right)^{p} d x\right)^{1 / q} \\
\leq C\|f\|_{p}^{1-p / q}\left(\|f\|_{p}^{p}\right)^{1 / q}=C\|f\|_{p}
\end{gathered}
$$

This establishes sufficiency of the conditions on $\gamma$ and $\beta$. To show necessity we assume the existence of the constant $C$ and consider $f \in L^{p}\left(\mathbb{R}^{n}\right)$ defined by $f(y)=$ $(1+|y|)^{-\frac{n}{p}-\epsilon}$ where $0<\epsilon<1$. Observe that for $|x|>1$ :

$$
\begin{array}{r}
T_{1, \gamma, \beta} f(x) \geq(1+|x|)^{\gamma} \int_{\frac{1}{2}|x| \leq|y| \leq|x|}(1+|y|)^{-\frac{n}{p}-\epsilon-\beta} d y \\
\geq C \min \left\{1,2^{\frac{n}{p}+\epsilon+\beta}\right\}(1+|x|)^{\gamma-\frac{n}{p}-\epsilon-\beta}|x|^{n} \\
\geq C \min \left\{1,2^{\frac{n}{p}+\epsilon+\beta}\right\} \min \left\{1,2^{\gamma-\frac{n}{p}-\epsilon-\beta}\right\}|x|^{\gamma-\frac{n}{p}-\epsilon-\beta+n} \\
\geq C|x|^{\gamma-\frac{n}{p}-\epsilon-\beta+n}
\end{array}
$$

where we used the boundedness of $\epsilon$ between 0 and 1 to make the constants inde- 
pendent of $\epsilon$. Certainly, we must have $\left\|T_{1, \gamma, \beta} f\right\|_{q}<\infty$ and so $\gamma-\frac{n}{p}-\epsilon-\beta+n<-\frac{n}{q}$ for every $0<\epsilon<1$ which implies $\gamma-\beta \leq-n\left(\frac{1}{p^{\prime}}+\frac{1}{q}\right)$. To establish the necessity of the condition on gamma we consider $f=\chi_{B(0,1)}$ and observe that for $|x|>1$ :

$T_{1, \gamma, \beta} f(x)=(1+|x|)^{\gamma} \int_{|y| \leq|x|} \frac{\chi_{B(0,1)}}{(1+|y|)^{\beta}} d y \geq(1+|x|)^{\gamma} \int_{|y| \leq 1} \frac{1}{(1+|y|)^{\beta}} d y=C(1+|x|)^{\gamma}$

Finally, the existence of $C$ forces $\gamma<-\frac{n}{q}$ as desired.

Obtaining the necessary bounds on $T_{2}$ will make use of the following sequence of definitions and propositions. These results are well known but for a brief development see section 2.8 in [4].

Definition 2.3: The Hardy-Littlewood maximal function $M f$ of a locally integrable function $f: \mathbb{R}^{n} \rightarrow \mathbb{R}$ is defined by:

$$
M f(x)=\sup _{r>0} r^{-n} \int_{B(x, r)}|f(y)| d y
$$

Proposition 2.4: If $1<p<\infty$ and $f \in L^{p}\left(\mathbb{R}^{n}\right)$ then $M f \in L^{p}\left(\mathbb{R}^{n}\right)$ and $\|M f\|_{p} \leq$ $C_{p, n}\|f\|$.

Definition 2.5: For $0<\alpha<n$ the Riesz potential of order $\alpha$ for each $f$ is given by:

$$
I_{\alpha} f(x)=\int_{\mathbb{R}^{n}} \frac{f(y)}{|x-y|^{n-\alpha}} d y
$$


Proposition 2.6: If $0<\delta<n, \rho>0$, and $f: \mathbb{R}^{n} \rightarrow \mathbb{R}$ is locally integrable then:

$$
\int_{|x-y| \leq \rho} \frac{f(y)}{|x-y|^{\delta}} d y \leq C_{n, \delta} \rho^{n-\delta} M f(x)
$$

The following is called the Sobolev inequality.

Proposition 2.7: Let $\alpha>0,1<p<\infty, p<\frac{n}{\alpha}$. Then there is a constant $C$, independent of $f$, such that:

$$
\left\|I_{\alpha}(f)\right\|_{p^{*}} \leq C\|f\|_{p}, \quad p^{*}=\frac{n p}{n-\alpha p}
$$

for all $f \in L^{p}\left(\mathbb{R}^{n}\right)$.

Now define:

$$
T_{2, \gamma, \lambda} f(x)=(1+|x|)^{\gamma} \int_{\frac{1}{2}|x| \leq|y| \leq 2|x|} \frac{f(y)}{(1+|x-y|)^{\lambda}} d y
$$

Lemma 2.8: Suppose that $q \geq p$. Then $\left\|T_{2,-\alpha-\beta, \lambda} f\right\|_{q} \leq C\|f\|_{p}$ if the following are satisfied:

$$
\begin{array}{r}
\alpha+\beta \geq 0 \\
\alpha+\beta+\lambda \geq n\left(\frac{1}{p^{\prime}}+\frac{1}{q}\right) \\
\alpha+\beta+\lambda=n\left(\frac{1}{p^{\prime}}+\frac{1}{q}\right) \Longrightarrow \lambda>0 \\
p=q, \alpha+\beta+\lambda=n \Longrightarrow \lambda<n
\end{array}
$$

Case 1. $\alpha+\beta+\lambda>n\left(\frac{1}{p^{\prime}}+\frac{1}{q}\right)$. Since $\alpha+\beta \geq 0$ and that $\frac{1}{2}|x| \leq|y| \leq 2|x| \Longrightarrow|x|>$ $C|x-y|$ we have that:

$$
T_{2,-\alpha-\beta, \lambda} f(x) \leq \int_{\mathbb{R}^{n}} \frac{f(y)}{(1+|x-y|)^{\alpha+\beta+\lambda}} d y
$$


Notice that this integral is a convolution of the functions $f$, and $g(x)=\frac{1}{(1+|x|)^{\alpha+\beta+\lambda}}$. Define $\frac{1}{r}=\frac{1}{p^{\prime}}+\frac{1}{q}$. Since $\frac{1}{q}+1=\frac{1}{p}+\frac{1}{r}$, Young's convolution inequality tells us that:

$$
\left\|T_{2,-\alpha-\beta, \lambda} f\right\|_{q} \leq\|f\|_{p}\left\|(1+|x|)^{-(\alpha+\beta+\lambda)}\right\|_{r} \leq C\|f\|_{p}
$$

where the last inequality follows from the fact that $(\alpha+\beta+\lambda) r>n$.

Case 2. $\alpha+\beta+\lambda=n\left(\frac{1}{p^{\prime}}+\frac{1}{q}\right)$, and $q>p$. In this case we get $\lambda<n$ for free since $\lambda \leq \alpha+\beta+\lambda=n\left(\frac{1}{p^{\prime}}+\frac{1}{q}\right)<n$. Also $\frac{1}{2}|x| \leq|y| \leq 2|x| \Longrightarrow|x-y| \leq 3|x| \Longrightarrow$ $(1+|x|)^{-\alpha-\beta} \leq C(1+|x-y|)^{-\alpha-\beta}$. Therefore:

$$
T_{2,-\alpha-\beta, \lambda} f(x) \leq C \int_{\mathbb{R}^{n}} \frac{f(y)}{(1+|x-y|)^{\alpha+\beta+\lambda}} d y \leq C \int_{\mathbb{R}^{n}} \frac{f(y)}{|x-y|^{n\left(\frac{1}{q}+\frac{1}{p^{\prime}}\right)}} d y
$$

Now just apply the Sobolev inequality with $\alpha=n-\frac{n}{q}-\frac{n}{p^{\prime}}$ to get the result.

Case 3. $\alpha+\beta+\lambda=n\left(\frac{1}{p^{\prime}}+\frac{1}{q}\right)$, and $q=p$. We have:

$$
T_{2,-\alpha-\beta, \lambda} f(x) \leq|x|^{\lambda-n} \int_{|x-y| \leq 3|x|} \frac{f(y)}{|x-y|^{\lambda}} \leq \operatorname{CMf}(x)
$$

where the last inequality follows from proposition 2.6. Now simply taking the $p$-norm and applying proposition 2.4 gives the result.

Define:

$$
T_{3, \gamma, \beta} f(x)=(1+|x|)^{\gamma} \int_{|y| \geq|x|} \frac{f(y)}{(1+|y|)^{\beta}} d y
$$

Lemma 2.9: Suppose that $1<p \leq q<\infty$. Then $T_{3, \gamma, \beta} f \in L^{q}\left(\mathbb{R}^{n}\right)$, and $\left\|T_{3, \gamma, \beta} f\right\|_{q} \leq$ $C\|f\|_{p}$ if and only if $\beta>\frac{n}{p^{\prime}}$, and $\gamma-\beta \leq-n\left(\frac{1}{q}+\frac{1}{p^{\prime}}\right)$.

We begin by proving sufficiency. 
Case 1. $p=q$. We have:

$$
\left\|T_{3, \gamma, \beta} f\right\|_{p}=\left(\int_{\mathbb{R}^{n}}\left(\int_{|y| \geq|x|} \frac{(1+|x|)^{\gamma}}{(1+|y|)^{\beta}} f(y) d y\right)^{p} d x\right)^{1 / p}
$$

We proceed identically to the $|y| \leq|x|$ case. After the step involving Fubini's theorem we obtain:

$$
\left\|T_{3, \gamma, \beta} f\right\|_{p} \leq C \int_{1}^{\infty} \int_{S^{n-1}}\left(\int_{0}^{\infty}\left(\frac{(1+s)^{\gamma}}{(1+s t)^{\beta}} f(s t \omega) s^{n} t^{n-1}\right)^{p} s^{n-1} d s\right)^{1 / p} d \omega d t
$$

Subcase 1. $\beta \geq n$. We claim that $\frac{(1+s)^{\gamma}}{(1+s t)^{\beta}} s^{n} \leq C t^{-n}$ whenever $t>1$. If $s>1$ we have:

$$
\frac{(1+s)^{\gamma}}{(1+s t)^{\beta}} s^{n} \leq \frac{(1+s)^{\beta-n}}{(1+s t)^{\beta}} s^{n} \leq C \frac{s^{\beta-n} s^{n}}{s^{\beta} t^{\beta}} \leq C t^{-n}
$$

And if $0 \leq s \leq 1$ then $(1+s)^{\gamma} \approx C$ so that:

$$
\frac{(1+s)^{\gamma}}{(1+s t)^{\beta}} s^{n} \leq \frac{C s^{n}}{(1+s t)^{\beta}} \leq \frac{C s^{n}}{(1+s t)^{n}} \leq \frac{C s^{n}}{s^{n} t^{n}}=C t^{-n}
$$

Now we proceed identically to the $|y| \leq|x|$ case and and apply Holder's inequality to the integral over $S^{n-1}$ before applying the previous estimate and making the substitution $s=\frac{1}{t} r$.

$$
\begin{gathered}
\int_{S^{n-1}}\left(\int_{0}^{\infty}\left(\frac{(1+s)^{\gamma}}{(1+s t)^{\beta}} f(s t \omega) s^{n} t^{n-1}\right)^{p} s^{n-1} d s\right)^{1 / p} d \omega \\
\leq C t^{-1}\left(\int_{S^{n-1}} \int_{0}^{\infty} f(s t \omega)^{p} s^{n-1} d s d \omega\right)^{1 / p} \\
\leq C t^{-1-\frac{n}{p}}\|f\|_{p}
\end{gathered}
$$

which ensures the integral over $t$ is finite giving $\left\|T_{3, \gamma, \beta} f\right\|_{p} \leq C\|f\|_{p}$ as desired. 
Subcase 2. $\frac{n}{p^{\prime}}<\beta<n$. If $t>1$ we have:

$$
\frac{(1+s)^{\gamma}}{(1+s t)^{\beta}} s^{n} \leq \frac{(1+s)^{\beta-n}}{(1+s t)^{\beta}} s^{n} \leq \frac{s^{\beta-n} s^{n}}{(1+s t)^{\beta}} \leq \frac{s^{\beta}}{s^{\beta} t^{\beta}}=t^{-\beta}
$$

We again use Holder's inequality, the previous estimate, and the substitution $s=\frac{1}{t} r$ :

$$
\begin{gathered}
\int_{S^{n-1}}\left(\int_{0}^{\infty}\left(\frac{(1+s)^{\gamma}}{(1+s t)^{\beta}} f(s t \omega) s^{n} t^{n-1}\right)^{p} s^{n-1} d s\right)^{1 / p} d \omega \\
\leq C t^{n-\beta-1}\left(\int_{S^{n-1}} \int_{0}^{\infty} f(s t \omega)^{p} s^{n-1} d s d \omega\right)^{1 / p} \\
\leq C t^{n-\beta-1-\frac{n}{p}}\|f\|_{p} \\
=C t^{\frac{n}{p^{\prime}}-\beta-1}\|f\|_{p}
\end{gathered}
$$

which will integrate over $t$ so that $\left\|T_{3, \gamma, \beta} f\right\|_{p} \leq C\|f\|_{p}$ as desired.

Case $q>p$. In this case we have:

$$
\begin{aligned}
& \left(T_{3, \gamma, \beta} f(x)\right)^{q}=(1+|x|)^{\gamma q}\left(\int_{|y| \geq|x|} \frac{f(y)}{(1+|y|)^{\beta}} d y\right)^{q-p}\left(\int_{|y| \geq|x|} \frac{f(y)}{(1+|y|)^{\beta}} d y\right)^{p} \\
= & (1+|x|)^{\gamma q+n p-\beta p}\left(\int_{|y| \geq|x|} \frac{f(y)}{(1+|y|)^{\beta}} d y\right)^{q-p}\left((1+|x|)^{\beta-n} \int_{|y| \geq|x|} \frac{f(y)}{(1+|y|)^{\beta}} d y\right)^{p} \\
\leq & (1+|x|)^{\gamma q+n p-\beta p}\left(\|f\|_{p}\left(\int_{|y| \geq|x|} \frac{1}{(1+|y|)^{\beta p^{\prime}}} d y\right)^{\frac{1}{p^{\prime}}}\right)^{q-p}\left((1+|x|)^{\beta-n} \int_{|y| \geq|x|} \frac{f(y)}{(1+|y|)^{\beta}} d y\right)^{p} \\
\leq & C(1+|x|)^{\gamma q+n p-\beta p}\|f\|_{p}^{q-p}\left(\int_{|x|}^{\infty}(1+r)^{n-1-\beta p^{\prime}} d r\right)^{\frac{q-p}{p^{\prime}}}\left((1+|x|)^{\beta-n} \int_{|y| \geq|x|} \frac{f(y)}{(1+|y|)^{\beta}} d y\right)^{p} \\
= & C(1+|x|)^{\gamma q+n p-\beta p}\|f\|_{p}^{q-p}\left((1+|x|)^{n-\beta p^{\prime}}\right)^{\frac{q-p}{p^{\prime}}}\left((1+|x|)^{\beta-n} \int_{|y| \geq|x|} \frac{f(y)}{(1+|y|)^{\beta}} d y\right)^{p} \\
= & C(1+|x|)^{\gamma q+n p+\frac{n}{p^{\prime}}(q-p)-\beta q}\|f\|_{p}^{q-p}\left((1+|x|)^{\beta-n} \int_{|y| \geq|x|} \frac{f(y)}{(1+|y|)^{\beta}} d y\right)^{p} \\
\leq & C\|f\|_{p}^{q-p}\left((1+|x|)^{\beta-n} \int_{|y| \geq|x|} \frac{f(y)}{(1+|y|)^{\beta}} d y\right)^{p}
\end{aligned}
$$


where we used Holder's inequality in the first inequality and some basic calculus. Now just integrate, use the $p=q$ case and raise to the $\frac{1}{q}$ to get the result.

Now we show that $\gamma-\beta \leq-n\left(\frac{1}{q}+\frac{1}{p^{\prime}}\right)$ is necessary. Assume that $\left\|T_{3, \gamma, \beta} f\right\|_{p} \leq C\|f\|_{p}$, fix $0<\epsilon<1$, and suppose that $f(x)=(1+|x|)^{-\frac{n}{p}-\epsilon}$. Note that $f \in L^{p}\left(\mathbb{R}^{n}\right)$ We have:

$$
\left\|T_{3, \gamma, \beta} f\right\|_{q}^{q}=\int_{\mathbb{R}^{n}}(1+|x|)^{\gamma q}\left(\int_{|y| \geq|x|}(1+|y|)^{-\frac{n}{p}-\epsilon-\beta} d y\right)^{q} d x
$$

Obviously the inner integral must be finite for almost all $x$ so we must have $\beta+\frac{n}{p}+\epsilon>$ $n \forall 0<\epsilon<1$. This forces $\beta \geq \frac{n}{p^{\prime}}$.

$$
\begin{gathered}
\geq \int_{\mathbb{R}^{n}}(1+|x|)^{\gamma q}\left(\int_{|x| \leq|y| \leq 2|x|}(1+|y|)^{-\frac{n}{p}-\epsilon-\beta} d y\right)^{q} d x \\
\geq \frac{1}{2^{\frac{n q}{p}}+\epsilon q+\beta q} \int_{\mathbb{R}^{n}}(1+|x|)^{\gamma q}\left(\int_{|x| \leq|y| \leq 2|x|}(1+|x|)^{-\frac{n}{p}-\epsilon-\beta} d y\right)^{q} d x \\
\geq \frac{C}{2^{\frac{n q}{p}+\epsilon q+\beta q}} \int_{|x|>1}(1+|x|)^{\gamma q-\frac{n q}{p}-\epsilon q-\beta q}|x|^{n q} d x \\
\geq \frac{C \min \left\{1,2^{\gamma q-\frac{n q}{p}-\epsilon q-\beta q}\right\}}{2^{\frac{n q}{p}}+\epsilon q+\beta q} \int_{|x|>1}|x|^{\gamma q-\frac{n q}{p}-\epsilon q-\beta q+n q} d x
\end{gathered}
$$

Since the constants are exponential functions of $\epsilon$ and $0<\epsilon<1$ they are bounded away from zero and therefore can be made independent of $\epsilon$. So we must have: $\gamma q-\frac{n q}{p}-\epsilon q-\beta q+n q<-n$. Letting $\epsilon$ go to zero and rearranging gives: $\gamma-\beta \leq$ $-n\left(\frac{1}{q}+\frac{1}{p^{\prime}}\right)$.

To show that $\beta=\frac{n}{p^{\prime}}$ is not allowed we will provide a specific function for which 
the desired inequality fails to hold when $\beta=\frac{n}{p^{\prime}}$. Consider a function of the form:

$$
f(x)=\sum_{k=1}^{\infty} C_{k} \chi_{\left\{2^{k-1} \leq|x| \leq 2^{k}\right\}}
$$

It will suffice to select $C_{k} \geq 0$ so that $f \in L_{p}\left(\mathbb{R}^{n}\right)$, but $T_{3, \gamma, \beta} f(x)=\infty$ on a set of non-zero measure. When $|x|<1$ we have $(1+|x|)^{\gamma} \approx 1$. Moreover, $|y| \geq 1 \Longrightarrow$ $(1+|y|)^{\beta} \approx|y|^{\beta}$ so that:

$$
\begin{gathered}
T_{3, \gamma, \beta} f(x) \geq C \int_{\{|y| \geq 1\}} \frac{f(y)}{(1+|y|)^{\beta}} d y \\
\geq C \sum_{k=1}^{\infty} C_{k} \int_{\left\{2^{k-1} \leq|y| \leq 2^{k}\right\}} \frac{1}{|y|^{\beta}} d y \\
\geq C \sum_{k=1}^{\infty} C_{k} 2^{k(n-\beta)}
\end{gathered}
$$

Now, $\|f\|_{p}^{p} \approx \sum_{k=1}^{\infty} C_{k}^{p} 2^{n k}$ so with $\beta=\frac{n}{p^{\prime}}$ we will be done if we can find $\left\{C_{k}\right\}$ so that $\sum_{k=1}^{\infty} C_{k}^{p} 2^{n k}<\infty$ but $\sum_{k=1}^{\infty} C_{k} 2^{k \frac{n}{p}}=\infty$. Choosing $C_{k}=\frac{1}{k 2^{\frac{n k}{p}}}$ gives this result.

As stated previously what we're ultimately trying to achieve are useful bounds on the norms of the convolution operator which we can use in our analysis of partial differential equations. In addition to being an important step in proving the necessity of the conditions on the boundedness of the integral operator of this chapter, the following lemma makes explicit the connection between the integral operator of this chapter and convolution inequalities of the form we wish to use.

Lemma 2.10: Suppose that the forward direction of the main theorem is true, i.e. that there is $C$ independent of $f$ such that $\|T f\|_{q} \leq C\|f\|_{p}$. Then constants indepen- 
dent of $f$ can be found so that:

$$
\begin{aligned}
& \|f * g\|_{-\alpha ; q} \leq C\|f\|_{\beta ; p}\|g\|_{\lambda ; \infty} \\
& \|f * g\|_{-\lambda ; 1} \leq C\|f\|_{\beta ; p}\|g\|_{\alpha ; q^{\prime}}
\end{aligned}
$$

Proof: To get the first inequality observe that:

$$
\begin{gathered}
(1+|x|)^{-\alpha} f * g(x)=(1+|x|)^{-\alpha} \int_{\mathbb{R}^{n}} f(y) g(x-y) d y \\
=\int_{\mathbb{R}^{n}} \frac{(1+|y|)^{\beta} f(y)(1+|x-y|)^{\lambda} g(x-y)}{(1+|x|)^{\alpha}(1+|x-y|)^{\lambda}(1+|y|)^{\beta}} d y \\
\leq\|g\|_{\lambda, \infty} \int_{\mathbb{R}^{n}} \frac{(1+|y|)^{\beta} f(y)}{(1+|x|)^{\alpha}(1+|x-y|)^{\lambda}(1+|y|)^{\beta}} d y \\
=\|g\|_{\lambda, \infty} T\left[(1+|x|)^{\beta} f(x)\right]
\end{gathered}
$$

Now just $q$-norm both sides and apply the hypothesis. The proof of the second inequality is virtually identical.

The following important lemma will be proved in the section on weighted $L^{p}$ space convolution inequalities. It will be obtained as a necessary condition on the inequalities in that section.

Lemma 2.11: If there is $C$ independent of $f$ and $g$ such that $\|f * g\|_{\theta ; p_{0}} \leq C\|f\|_{\sigma ; p_{1}}\|g\|_{\gamma ; p_{2}}$ then $\sigma+\gamma \geq 0$.

Since $|y| \leq \frac{1}{2}|x| \Longrightarrow|x-y| \approx|x|,|y| \geq 2|x| \Longrightarrow|x-y| \approx|y|$, and $\frac{1}{2}|x| \leq|y| \leq 2|x| \Longrightarrow|x| \approx|y|$ we have that:

$$
T f(x) \approx T_{1,-\alpha-\lambda, \beta} f(x)+T_{2,-\alpha-\beta, \lambda} f(x)+T_{3,-\alpha, \lambda+\beta} f(x)
$$


so that the forward direction of the main theorem follows immediately from Lemmas 2.2, 2.8, and 2.9. Lemmas 2.2, 2.8, and 2.9 also prove some of the necessary conditions with the necessity of $\alpha+\beta \geq 0$ following from Lemma 2.10 and 2.11.

\subsection{A CLARIFICATION}

It's useful to take a moment to ensure there is no circular reasoning happening in this chapter and the next. The forward direction of Theorem 2.1 will be used to obtain convolution inequalities of the form seen in Lemma 2.11 in the next chapter. At that point we will prove Lemma 2.11 without relying on the backward direction of Theorem 2.1 so we may use 2.11 in this chapter for the backward direction of Theorem 2.1 . 


\section{CHAPTER 3}

\section{CONVOLUTION INEQUALITIES IN WEIGHTED $L_{p}$ SPACES}

\subsection{A MULTILINEAR INTERPOLATION THEOREM}

In this chapter we will use the inequalities obtained from the previous chapter and the techniques of interpolation of operators in deriving more general convolution inequalities that will help us in our analysis of PDEs. The main interpolation theorem we will use is a multilinear version of the Riesz-Thorin interpolation theorem that also allows for change of measures. We present a proof whose inspiration is due to two passing hints in the exercises of [5]. In particular, see Exercises 12 and 13 in Section 1.6. The structure of the proof will be similar to the classic one and therefore make use of the Hadamard three lines lemma stated below:

Lemma 3.1 (Hadamard Three Lines Lemma) Let $F(z)$ be a complex valued func-

tion defined on $0 \leq \operatorname{Re}(z) \leq 1$. For $0 \leq \theta \leq 1$ define $M_{\theta}=\sup _{y \in \mathbb{R}}|F(\theta+i y)|$. If $F$ is bounded and continuous for $0 \leq \operatorname{Re}(z) \leq 1$ and analytic on $0<\operatorname{Re}(z)<1$ then for all $0 \leq \theta \leq 1$ we have $M_{\theta} \leq M_{0}^{1-\theta} M_{1}^{\theta}$.

Theorem 3.2. Suppose that $0<\theta<1,1<p, q, r<\infty$ and $\forall 0 \leq j \leq l$ :

$$
\begin{aligned}
& \frac{1}{r_{j}}=\frac{(1-\theta)}{p_{j}}+\frac{\theta}{q_{j}} \\
& \frac{1}{r}=\frac{(1-\theta)}{p}+\frac{\theta}{q}
\end{aligned}
$$

and that for suitable weight functions $u_{i}(x), v_{i}(x)$, and $w_{i}(x)$ :

$$
T: L_{p_{1}, u_{1}} \times \ldots \times L_{p_{l}, u_{l}}\left(\mathbb{R}^{n}\right) \rightarrow L_{p, u}\left(\mathbb{R}^{n}\right)
$$




$$
T: L_{q_{1}, v_{1}} \times \ldots \times L_{q_{l}, u_{l}}\left(\mathbb{R}^{n}\right) \rightarrow L_{q, v}\left(\mathbb{R}^{n}\right)
$$

with norms $M_{0}$ and $M_{1}$ respectively (i.e. $T$ is an l-linear operator between two pairs of spaces) then:

$$
T: L_{r_{1}, w_{1}} \times \ldots \times L_{r_{l}, w_{l}}\left(\mathbb{R}^{n}\right) \rightarrow L_{r, w}\left(\mathbb{R}^{n}\right)
$$

with norm $M \leq M_{0}^{1-\theta} M_{1}^{\theta}$ and $u_{j}, w_{j}, v_{j}, u, v, w$ defined analogously to $w=u^{r_{j} \frac{(1-\theta)}{p_{j}}} v^{r_{j} \frac{\theta}{q_{j}}}$.

Proof. We will sometimes use $y$ in place of $x$ to emphasize that the quantity is associated with the output spaces above. Define the following:

$$
\langle h, g\rangle=\int_{\mathbb{R}^{n}} h(y) g(y) d w
$$

Let $r^{\prime}$ denote the Hölder conjugate of $r$. Using the fact that:

$$
\|h\|_{r, w}=\sup _{\|g\|_{r^{\prime}, w}=1}|\langle h, g\rangle|
$$

we get:

$$
M=\sup _{\left\|f_{j}\right\|_{p_{j}, w_{j}=1}}\left\|T\left(f_{1}, \ldots, f_{l}\right)\right\|_{r, w}=\sup _{\left\|f_{j}\right\|_{p_{j}, w_{j}}=\|g\|_{r^{\prime}, w}=1}\left|\left\langle T\left(f_{1}, \ldots, f_{l}\right), g\right\rangle\right|
$$

Due to the density of bounded compactly supported functions in $L_{p}$ for suitably weighted Lesbegue spaces, it will suffice to prove that if $f_{i}, g$ are compactly supported simple functions with $\left\|f_{j}\right\|_{p_{j}, w_{j}}=\|g\|_{r^{\prime}, w}=1$ we get:

$$
\left|\left\langle T\left(f_{1}, \ldots, f_{l}\right), g\right\rangle\right| \leq M_{0}^{1-\theta} M_{1}^{\theta}
$$

For $0 \leq \operatorname{Re}(z) \leq 1$ define $\forall 1 \leq j \leq l$ :

$$
\frac{1}{r_{j}(z)}=\frac{(1-z)}{p_{j}}+\frac{z}{q_{j}}
$$




$$
\frac{1}{r^{\prime}(z)}=\frac{(1-z)}{p^{\prime}}+\frac{z}{q^{\prime}}
$$

Let $\left\{u_{j}^{(k)}(x)\right\},\left\{v_{j}^{(k)}(x)\right\},\left\{u^{(k)}(x)\right\},\left\{v^{(k)}(x)\right\}$ be increasing sequences of simple functions converging pointwise to $u_{i}(x), v_{i}(x), u(x), v(x)$ respectively. Define $w^{(k)}(x)$, $u^{(k)}(x), v^{(k)}(x)$ analogously to $w$. Then define:

$$
\begin{gathered}
\phi_{j}^{(k)}(x, z)=\left|f_{j}(x)\right|^{\frac{r_{j}}{r_{j}(z)}} \frac{f_{j}(x)}{\left|f_{i}(x)\right|} w_{j}^{(k)}(x)^{\frac{1}{r_{j}(z)}} u_{i}^{(k)}(x)^{-\frac{1-z}{p_{j}}} v_{j}^{(k)}(x)^{-\frac{z}{q_{i}}} \\
\psi^{(k)}(y, z)=|g(y)|^{\frac{r^{\prime}}{r^{\prime}(z)}} \frac{g(y)}{|g(y)|} w^{(k)}(y)^{\frac{1}{r^{\prime}(z)}} u^{(k)}(y)^{-\frac{1-z}{p^{\prime}}} v^{(k)}(y)^{-\frac{z}{q^{\prime}}}
\end{gathered}
$$

with the understanding that if any of $\left|f_{j}(x)\right|,|g(y)|$ are zero we say the corresponding $\phi_{j}^{(k)}(x, z), \psi^{(k)}(y, z)$ is zero as well. In the calculations that follow we will make frequent use of the fact that if $a \geq 0$ and $x, y \in \mathbb{R}$ then $\left|a^{x+i y}\right|=a^{x}$. Define the following function:

$$
F_{k}(z)=\left\langle T\left(\phi_{1}^{(k)}(z), \ldots, \phi_{l}^{(k)}(z)\right), \psi^{(k)}(z)\right\rangle
$$

We will show that $F_{k}$ satisfies the hypotheses of the Three Lines Lemma. Note that $\phi_{i}^{(k)}, \psi^{(k)}$ are compactly supported simple functions multiplied by simple functions. Therefore $T\left(\phi_{1}^{(k)}(z), \ldots, \phi_{l}^{(k)}(z)\right) \in L_{q, v}$, and $\psi^{(k)}(z) \in L_{q^{\prime}, v}$ for all $k, z$. To see that $F_{k}$ is analytic on $0<\operatorname{Re}(z)<1$ and continuous on $0 \leq \operatorname{Re}(z) \leq 1 \forall k$ check that the linearity of $T$ allows us to write $F_{k}(z)$ as a function of $z$ as linear combinations of real numbers raised to a complex power.

In the calculations that follow we will make frequent use of the fact that for $a \in \mathbb{R}^{+}$, $x, y \in \mathbb{R}$ we have $\left|a^{x+i y}\right|=a^{x}$. For $t \in \mathbb{R}$ and $\forall 1 \leq j \leq l$ we have:

$$
\frac{1}{r_{j}(i t)}=\frac{1}{p_{j}}-i\left(\frac{t}{p_{j}}-\frac{t}{q_{j}}\right)
$$


so that:

$$
\left|\phi_{j}^{(k)}(x, i t)\right|=\left|f_{i}(x)\right|^{\frac{r_{j}}{p_{j}}} w_{j}^{(k)}(x)^{\frac{1}{p_{j}}} u_{j}^{(k)}(x)^{-\frac{1}{q_{j}}}
$$

We claim that $\lim _{k \rightarrow \infty}\left\|\phi_{k}(i t)\right\|_{p_{j}, u_{j}}=1$. Observe:

$$
\begin{gathered}
\lim _{k \rightarrow \infty}\left\|\phi_{j}^{(k)}(i t)\right\|_{p_{j}, u_{j}}^{p_{j}}=\lim _{k \rightarrow \infty} \int_{\mathbb{R}^{n}}\left|\phi_{j}^{(k)}(x, i t)\right|^{p_{j}} u_{j}(x) d x \\
=\lim _{k \rightarrow \infty} \int_{\mathbb{R}^{n}}\left|f_{j}(x)\right|^{r_{j}} w^{(k)}(x) u_{j}^{(k)}(x)^{-1} u_{j}(x) d x
\end{gathered}
$$

Notice that due to the the compact support of $f$ and the fact that all functions in sight are simple, the following function is a majorant for the integrand:

$$
\left|f_{j}(x)\right|^{r_{j}} w(x) u_{j}^{(1)}(x)^{-1} u_{j}(x)
$$

so that we can continue the previous sequence of equalities with:

$$
\begin{gathered}
=\int_{\mathbb{R}^{n}} \lim _{k \rightarrow \infty}\left|f_{j}(x)\right|^{r_{j}} w^{(k)}(x) u_{j}^{(k)}(x)^{-1} u_{j}(x) d x \\
=\int_{\mathbb{R}^{n}}\left|f_{j}(x)\right|^{r_{j}} w(x) d x=\|f\|_{r_{j}, w}^{r_{j}}=1
\end{gathered}
$$

Similarly since:

$$
\frac{1}{r_{j}(1+i t)}=\frac{1}{q_{j}}+i\left(\frac{t}{q_{j}}-\frac{t}{p_{j}}\right)
$$

we get $\lim _{k \rightarrow \infty}\left\|\phi_{j}^{(k)}(1+i t)\right\|_{q_{j}, v_{j}}=1$. Similar arguments give:

$$
\lim _{k \rightarrow \infty}\left\|\psi^{(k)}(i t)\right\|_{p^{\prime}, u}=\lim _{k \rightarrow \infty}\left\|\psi^{(k)}(1+i t)\right\|_{q^{\prime}, v}=1
$$

So that for any $\epsilon>0$ we can select $k$ and use Hölder's inequality to bound $F_{k}$ at the edges of the strip:

$$
\left|F_{k}(i t)\right| \leq\left\|T\left(\phi_{1}^{(k)}(i t), \ldots, \phi_{l}^{(k)}(i t)\right)\right\|_{p, u}\left\|\psi^{(k)}(i t)\right\|_{p^{\prime}, u} \leq M_{0}+\epsilon
$$




$$
\left|F_{k}(1+i t)\right| \leq\left\|T\left(\phi_{1}^{(k)}(1+i t), \ldots, \phi_{l}^{(k)}(1+i t)\right)\right\|_{q, v}\left\|\psi^{(k)}(1+i t)\right\|_{q^{\prime}, v} \leq M_{1}+\epsilon
$$

so that $\forall t \in \mathbb{R}$ and $0<\theta<1$ the three lines lemma gives us:

$$
\left|F_{k}(\theta+i t)\right| \leq M_{0}^{1-\theta} M_{1}^{\theta}
$$

and in particular for $t=0$ we get that

$$
\left|F_{k}(\theta)\right|=\left|\left\langle T\left(f_{1}, \ldots, f_{l}\right), g\right\rangle\right|=\leq\left(M_{0}+\epsilon\right)^{1-\theta}\left(M_{1}+\epsilon\right)^{\theta}
$$

so that by taking suprema over all such functions $f_{j}$ and $g$, and letting $\epsilon$ go to zero we get:

$$
M \leq M_{0}^{1-\theta} M_{1}^{\theta}
$$

\subsection{CONVOLUTION INEQUALITIES}

One well known convolution inequality we will make use of is Young's Inequality presented below along with the lesser known Peetre's Inequality.

Theorem 3.3: (Young's Inequality) If $f \in L^{p}\left(\mathbb{R}^{n}\right)$ and $g \in L^{q}\left(\mathbb{R}^{n}\right), p, q, r \geq 1$, and $1+\frac{1}{r}=\frac{1}{p}+\frac{1}{q}$ then $f * g \in L^{r}\left(\mathbb{R}^{n}\right)$ and:

$$
\|f * g\|_{r} \leq\|f\|_{p}\|g\|_{q}
$$

Theorem 3.4: (Peetre's Inequality) If $t \in \mathbb{R}$ and $x, y \in \mathbb{R}^{n}$, then:

$$
\left(\frac{1+|x|^{2}}{1+|y|^{2}}\right)^{t} \leq 2^{|t|}\left(1+|x-y|^{2}\right)^{|t|}
$$


Our methods for obtaining general convolution inequalities will be to apply the multilinear interpolation theorem to the bilinear convolution operator. For this we will need two convolution inequalities that satisfy the hypotheses of theorem 3.2 to interpolate between. One will be obtained via Young's inequality and the other will be obtained using the convolution inequality derived using our version of the Stein-Weiss inequality in Chapter 2. We will divide our sufficient conditions on the convolution inequality in the weighted $L^{p}$ space over three portions.

Theorem 3.5: There exists $C$ independent of $f$ and $g$ so that:

$$
\|f * g\|_{\theta ; a} \leq C\|f\|_{\gamma ; b}\|g\|_{\sigma ; c}
$$

whenever the following holds (or the identical set of conditions with the roles of $(\gamma ; b)$ and $(\sigma ; c)$ switched):
i) $0<1+\frac{1}{a}-\frac{1}{b}-\frac{1}{c}<1$
ii) $1<b \leq a<\infty, c<\infty$
iii) $\gamma+\sigma-\theta \geq n+\frac{n}{a}-\frac{n}{b}-\frac{n}{c}$
iv) $\gamma-\theta \geq 0$
v) $\sigma-\theta, \gamma+\sigma>0$
vi) $2 \sigma+\gamma-\theta>n+\frac{n}{a}-\frac{n}{b}-\frac{n}{c}$
vii) $\sigma-\theta>\frac{n}{a}-\frac{n}{c}$
viii) $\gamma+\sigma>n-\frac{n}{b}-\frac{n}{c}$
ix) $a=b$ and $\gamma+\sigma-\theta=n-\frac{n}{c} \Longrightarrow \sigma<n-\frac{n}{c}$
x) $a=b \Longrightarrow 1<c<\infty$

For easy reference, the following is a restatement of a portion of lemma 2.10 from the previous section.

Proposition 3.6: There are constants $C_{1}$ and $C_{2}$ independent of $f$ and $g$ such that

$$
\|f * g\|_{-\alpha ; q} \leq C_{1}\|f\|_{\beta ; p}\|g\|_{\lambda ; \infty}
$$




$$
\|f * g\|_{-\lambda ; 1} \leq C_{2}\|f\|_{\beta ; p}\|g\|_{\alpha ; q^{\prime}}
$$

whenever the following holds:

a) $1<p \leq q<\infty$

b) $\alpha+\lambda>\frac{n}{q}$

c) $\beta+\lambda>\frac{n}{p^{\prime}}$

d) $\alpha+\beta+\lambda \geq \frac{n}{p^{\prime}}+\frac{n}{q}$

e) $\alpha+\beta \geq 0$

f) $\alpha+\beta+\lambda=n$ and $p=q \Longrightarrow \lambda<n$

Proof of Theorem 3.5: If $a=b$ choose $t=1+\frac{1}{a}-\frac{1}{b}-\frac{1}{c}=1-\frac{1}{c}$. If $a>b$ choose $1+\frac{1}{a}-\frac{1}{b}-\frac{1}{c}<t<1-\frac{1}{c}$. The hypotheses imply that we may select $p^{\prime}>1$ so that:

$$
\max \left\{n+\frac{n}{a}-\frac{n}{b}-\frac{n}{c}-(\sigma-\theta), n-\frac{n}{b}-\frac{n}{c}, 0\right\}<\frac{n t}{p^{\prime}}<\min \left\{\gamma+\sigma, n-\frac{n}{b}, n+\frac{n}{a}-\frac{n}{b}-\frac{n}{c}\right\}
$$

Choose q so that $\frac{n t}{q}=n+\frac{n}{a}-\frac{n}{b}-\frac{n}{c}-\frac{n t}{p^{\prime}}$. Then q and p' satisfy:

$$
1+\frac{1}{a}-\frac{1}{b}-\frac{1}{c}=t\left(\frac{1}{p^{\prime}}+\frac{1}{q}\right)
$$

The choice of $p^{\prime}$ guarantees that $q>0$ and choice of $t$ combined with (9) implies that $\frac{1}{p^{\prime}}+\frac{1}{q} \leq 1$. Hence $1<p \leq q<\infty$. Define indices $\alpha=-\frac{\theta}{t}, \beta=\frac{\gamma}{t}, \lambda=\frac{\sigma}{t}$. It is readily shown that these indices satisfy a) through e) of proposition 3.3. When $a>b$ the choice of $t$ and (9) forces $\frac{1}{p^{\prime}}+\frac{1}{q}<1$ and so $p<q$ in this case, giving f), satisfied vacuously. In case $a=b$ our choice of $t$ and (9) gives us $\left(\frac{1}{p^{\prime}}+\frac{1}{q}\right)=1$ and so $q=p$. If $\gamma+\sigma-\theta>n+\frac{n}{a}-\frac{n}{b}-\frac{n}{c}=n-\frac{n}{c}$ then $\alpha+\beta+\lambda>n$ and so f) is again satisfied vacuously. If $\gamma+\sigma-\theta=n+\frac{n}{a}-\frac{n}{b}-\frac{n}{c}=n-\frac{n}{c}$ then $\alpha+\beta+\lambda=n$ and so we must use ix) to deduce that $\lambda<n$ and conclude that $\mathrm{f}$ ) is satisfied in this case as well. We 
apply proposition 3.6 to obtain:

$$
\|f * g\|_{\frac{\theta}{t} ; q} \leq C\|f\|_{\frac{\gamma}{t} ; p}\|g\|_{\frac{\sigma}{t} ; \infty}
$$

Define indices $r_{1}, r_{2}, r_{3}$ :

$$
r_{1}=\frac{1-t}{\frac{1}{a}-\frac{t}{q}}, r_{2}=\frac{1-t}{\frac{1}{b}-\frac{t}{p}}, r_{3}=\frac{1-t}{\frac{1}{c}}
$$

The choice of $t, p$, and $q$ guarantees that $r_{1}, r_{2}, r_{3} \geq 1$ and some algebra shows that $1+\frac{1}{r_{1}}=\frac{1}{r_{2}}+\frac{1}{r_{3}}$ so we may apply Young's Inequality to obtain:

$$
\|f * g\|_{0 ; r_{1}} \leq C\|f\|_{0 ; r_{2}}\|g\|_{0 ; r_{3}}
$$

It is clear that:

$$
\frac{1}{a}=\frac{t}{q}+\frac{1-t}{r_{1}}, \frac{1}{b}=\frac{t}{p}+\frac{1-t}{r_{2}}, \frac{1}{c}=\frac{t}{\infty}+\frac{1-t}{r_{3}}
$$

It's readily checked that with weights of the form $w(x)=(1+|x|)^{\alpha p}$ this is sufficient for the conditions of the main interpolation theorem of this chapter to be satisfied. So we may interpolate between (10) and (11) using theorem 3.2 to get the result.

Theorem 3.7: There exists $C$ independent of $f$ and $g$ such that:

$$
\|f * g\|_{\theta ; a} \leq C\|f\|_{\gamma ; b}\|g\|_{\sigma ; c}
$$

whenever the following holds:

i) $0<1+\frac{1}{a}-\frac{1}{b}-\frac{1}{c} \leq \frac{1}{a}$

ii) $b, c>1$

iii) $\gamma+\sigma-\theta \geq n+\frac{n}{a}-\frac{n}{b}-\frac{n}{c}$ 
iv) $\gamma+\sigma-2 \theta>n+\frac{n}{a}-\frac{n}{b}-\frac{n}{c}$

v) $\gamma+\sigma \geq 0$

vi) $\sigma-\theta, \gamma-\theta>0$

vii) $\sigma-\theta>\frac{n}{a}-\frac{n}{c}$

viii) $\gamma-\theta>\frac{n}{a}-\frac{n}{b}$

ix) $1=\frac{1}{b}+\frac{1}{c}$ and $\sigma+\gamma-\theta=\frac{n}{a} \Longrightarrow \theta>-\frac{n}{a}$

The case where the right half of condition i) is equality is not trivial but is similar to the following and therefore left to the reader.

Proof of Theorem 3.7 Suppose i) through ix) and select $1+\frac{1}{a}-\frac{1}{b}-\frac{1}{c}<t<\frac{1}{a}$. The hypotheses imply that if $a>1$ :

$\max \left\{n+\frac{n}{a}-\frac{n}{b}-\frac{n}{c}-(\sigma-\theta), \frac{n}{a}-\frac{n}{b}, 0\right\}<\min \left\{\gamma-\theta, n-\frac{n}{b}, n+\frac{n}{a}-\frac{n}{b}-\frac{n}{c}\right\}<n t$ and, if $a=1$ :

$$
\max \left\{n+\frac{n}{a}-\frac{n}{b}-\frac{n}{c}-(\sigma-\theta), 0\right\}<n-\frac{n}{b}<\min \left\{\gamma-\theta, n+\frac{n}{a}-\frac{n}{b}-\frac{n}{c}\right\}<n t
$$

So we may select $p^{\prime}>1$ so the following are simultaneously satisfied:

$$
\begin{gathered}
\frac{n}{a}-\frac{n}{b} \leq \frac{n t}{p^{\prime}} \leq n-\frac{n}{b} \\
\frac{n t}{p^{\prime}}<n+\frac{n}{a}-\frac{n}{b}-\frac{n}{c} \\
\gamma-\theta>\frac{n t}{p^{\prime}}>n+\frac{n}{a}-\frac{n}{b}-\frac{n}{c}-(\sigma-\theta)
\end{gathered}
$$

Now select $q$ so that $\frac{n t}{q}=n+\frac{n}{a}-\frac{n}{b}-\frac{n}{c}-\frac{n t}{p^{\prime}}$. Together $p^{\prime}$ and $q$ satisfy:

$$
t\left(\frac{1}{p^{\prime}}+\frac{1}{q}\right)=1+\frac{1}{a}-\frac{1}{b}-\frac{1}{c}
$$


By (14), $q$ is positive and so $t>1+\frac{1}{a}-\frac{1}{b}-\frac{1}{c}$ combined with (16) forces $\frac{1}{p^{\prime}}+\frac{1}{q}<1$ and hence $1<p<q<\infty$. It is readily checked that the stated hypotheses and (16) imply that the indices $\alpha=\frac{\sigma}{t}, \beta=\frac{\gamma}{t}$, and $\lambda=\frac{-\theta}{t}$ satisfy the hypotheses of Proposition 3.6 and so we conclude:

$$
\|f * g\|_{\frac{\theta}{t} ; 1} \leq C\|f\|_{\frac{\gamma}{t} ; p}\|g\|_{\frac{\sigma}{t} ; q^{\prime}}
$$

Define indices $r_{1}, r_{2}, r_{3}$ (We say they are $\infty$ if the denominators are zero):

$$
r_{1}=\frac{1-t}{\frac{1}{a}-t}, r_{2}=\frac{1-t}{\frac{1}{b}-\frac{t}{p}}, r_{3}=\frac{1-t}{\frac{1}{c}-\frac{t}{q^{\prime}}}
$$

Some algebra shows that $1+\frac{1}{r_{1}}=\frac{1}{r_{2}}+\frac{1}{r_{3}}$ and choice of $t$ and (13) implies that $r_{1}, r_{2}, r_{3} \geq 1$, so we may apply Young's Inequality to conclude:

$$
\|f * g\|_{0 ; r_{1}} \leq C\|f\|_{0 ; r_{2}}\|g\|_{0 ; r_{3}}
$$

It is clear that:

$$
\frac{1}{a}=\frac{t}{1}+\frac{1-t}{r_{1}}, \frac{1}{b}=\frac{t}{p}+\frac{1-t}{r_{2}}, \frac{1}{c}=\frac{t}{q^{\prime}}+\frac{1-t}{r_{3}}
$$

So we may interpolate between (17) and (18) to get the result.

The following theorem is a generalization of Young's Inequality to weights of the form $(1+|x|)^{\alpha}$.

Theorem 3.8: There is a constant $C$ independent of the functions $f$ and $g$ such that:

$$
\|f * g\|_{\theta ; a} \leq C\|f\|_{\gamma ; b}\|g\|_{\sigma ; c}
$$

whenever the following hold: 

i) $1+\frac{1}{a}-\frac{1}{b}-\frac{1}{c}=0$
ii) $\theta \leq \min \{\gamma, \sigma\}$
iii) $\gamma+\sigma \geq 0$

We will see near the end of the chapter that conditions ii) and iii) are necessary for the general convolution inequality. Therefore Theorem 3.5 fully characterizes the boundary case $1+\frac{1}{a}-\frac{1}{b}-\frac{1}{c}=0$.

Lemma 3.9: Suppose that $1+\frac{1}{a}-\frac{1}{b}-\frac{1}{c}=0$. Then for all $\theta \in \mathbb{R}$ we have that there is a constant $C$ independent of $f$ and $g$ so that:

$$
\|f * g\|_{\theta ; a} \leq C\|f\|_{|\theta| ; b}\|g\|_{\theta ; c}
$$

(Proof of Lemma 3.9) WLOG let's assume $f, g \geq 0$. We will make direct use of Peetre's and Young's Inequalities.

$$
\begin{aligned}
& \|f * g\|_{\theta, a}=\left(\int|f * g(x)|^{a}(1+|x|)^{\theta a} d x\right)^{\frac{1}{a}} \\
\leq & \left(\int\left(\int|f(y) g(x-y)| d y\right)^{a}(1+|x|)^{\theta a} d x\right)^{\frac{1}{a}} \\
= & \left(\int\left(\int|f(y) g(x-y)|(1+|x|)^{\theta} d y\right)^{a} d x\right)^{\frac{1}{a}}
\end{aligned}
$$

so that Peetre's Inequality gives us:

$$
\begin{gathered}
\|f * g\|_{\theta, a} \leq C\left(\int\left(\int\left|f(y)(1+|y|)^{\theta} g(x-y)\right|(1+|x-y|)^{|\theta|} d y\right)^{a} d x\right)^{\frac{1}{a}} \\
=C\left(\int f \cdot(1+|x|)^{\theta} * g \cdot(1+|x|)^{|\theta| a} d x\right)^{\frac{1}{a}} \\
=C\left\|f \cdot(1+|x|)^{\theta} * g \cdot(1+|x|)^{|\theta|}\right\|_{a}
\end{gathered}
$$




$$
\leq C\|f\|_{\theta, b}\|g\|_{|\theta|, r}
$$

where the last inequality follows from Young's Convolution Inequality.

(Proof of Theorem 3.8) Without loss of generality assume $\gamma \geq \sigma$. Then $\gamma \geq|\sigma|$ and so we apply Lemma 3.9:

$$
\|f * g\|_{\theta ; a} \leq\|f * g\|_{\sigma ; a} \leq C\|f\|_{|\sigma| ; b}\|g\|_{\sigma ; c} \leq C\|f\|_{\gamma ; b}\|g\|_{\sigma ; c}
$$

\subsection{NECESSARY CONDITIONS}

Now we find necessary conditions for the existence of a constant $C$ independent of the functions $f$, and $g$ so that:

$$
\|f * g\|_{\theta, p} \leq C\|f\|_{\gamma, p_{0}}\|g\|_{\sigma, p_{1}}
$$

We will see that in an important subcase the necessary conditions meet necessity up to boundary cases. Our methods will involve assuming the existence of such a constant and selecting appropriate choices for $f$ and $g$. To this end observe that if $\tau$, $\rho>0$ we have that:

$$
\begin{array}{r}
\tau^{n} \chi_{B_{\left(x_{0}+y_{0}, \rho\right)}}(x)=C \int_{\mathbb{R}^{n}} \chi_{B_{\left(y_{0}, \tau\right)}}(x-y) \chi_{B_{\left(x_{0}+y_{0}, \rho\right)}}(x) d y \\
=C \int_{\mathbb{R}^{n}} \chi_{B_{\left(x_{0}, \tau+\rho\right)}}(y) \chi_{B_{\left(y_{0}, \tau\right)}}(x-y) \chi_{B_{\left(x_{0}+y_{0}, \rho\right)}}(x) d y \\
\leq C \int_{\mathbb{R}^{n}} \chi_{B_{\left(x_{0}, \tau+\rho\right)}}(y) \chi_{B_{\left(y_{0}, \tau\right)}}(x-y) d y
\end{array}
$$

To obtain the second equality above we observed that:

$$
\left\{y:\left|x-y-y_{0}\right|<\tau\right\} \cap\left\{y:\left|x_{0}+y_{0}-x\right|<\rho\right\} \subset\left\{y:\left|x_{0}-y\right|<\tau+\rho\right\}
$$


The last integral is of the form $f * g(x)$, which motivates choosing $f(x)=\chi_{B_{\left(x_{0}, \tau+\rho\right)}}(x)$ and $g(x)=\chi_{B_{\left(y_{0}, \tau\right)}}(x)$. The hypothesis tells us there is a constant $C$ independent of $\tau, \rho, x_{0}$, and $y_{0}$ so that:

$$
\tau^{n}\left\|\chi_{B_{\left(x_{0}+y_{0}, \rho\right)}}\right\|_{\theta, p} \leq C\left\|\chi_{B_{\left(x_{0}, \rho+\tau\right)}}\right\|_{\gamma, p_{0}}\left\|\chi_{B_{\left(y_{0}, \tau\right)}}\right\|_{\sigma, p_{1}}
$$

Our task now is to select appropriate values for these parameters in order to obtain necessary conditions on $p, p_{0}, p_{1}, \theta, \gamma$, and $\sigma$. To this end we observe by direct calculation that in general $\left\|\chi_{B_{(\alpha, \rho)}}\right\|_{\alpha, p} \approx\left|x_{0}\right|^{\alpha} \rho^{\frac{n}{p}}$ when $\left|x_{0}\right| \geq 2$ and $\rho<\frac{1}{2}\left|x_{0}\right|$ and then impose the following restrictions:

$$
\rho<\frac{1}{2}\left|x_{0}+y_{0}\right|, \quad \rho+\tau<\left|x_{0}\right|, \quad \tau<\left|y_{0}\right|, \quad\left|x_{0}+y_{0}\right| \geq 2, \quad\left|x_{0}\right| \geq 2, \quad\left|y_{0}\right| \geq 2
$$

so that the convolution inequality becomes:

$$
\tau^{n} \rho^{\frac{n}{p}}\left|x_{0}+y_{0}\right|^{\theta} \leq C(\rho+\tau)^{\frac{n}{p_{0}}}\left|x_{0}\right|^{\gamma} \tau^{\frac{n}{p_{1}}}\left|y_{0}\right|^{\sigma}
$$

The restrictions on the parameters allow for $\left|x_{0}\right|=\left|y_{0}\right|=\left|x_{0}+y_{0}\right|=2$ and $\rho=\tau$ so that (7) reduces to:

$$
\rho^{n+\frac{n}{p}} \leq C \rho^{\frac{n}{p_{0}}+\frac{n}{p_{1}}}
$$

The constant $C$ must be independent of $\rho$ and this inequality must continue to hold for small $\rho$ which forces:

$$
1+\frac{1}{p}-\frac{1}{p_{0}}-\frac{1}{p_{1}} \geq 0
$$

Now take $\left|x_{0}\right|=\left|y_{0}\right|$ to be large with $\left|x_{0}+y_{0}\right|=2$ and let $\rho=\tau$ be fixed and small so that the restrictions (10) are still satisfied. Then using (7) we see that there must be a constant $C$ independent of $\left|x_{0}\right|$ so that $C \leq\left|x_{0}\right|^{\gamma+\sigma}$. Since we are free to take 
$\left|x_{0}\right|$ as large as we like:

$$
\gamma+\sigma \geq 0
$$

Remark 3.10: Obtaining (23) establishes Lemma 2.11 in the previous chapter.

Now suppose that $\left|x_{0}+y_{0}\right|=\left|x_{0}\right|, y_{0}=2, \tau=\frac{1}{2}$ and $\rho$ is fixed. Then (7) again tells us there must be a constant $C$ independent of $\left|x_{0}\right|$ so that $\left|x_{0}\right|^{\theta} \leq C\left|x_{0}\right|^{\gamma}$. Since we're still free to take $\left|x_{0}\right|$ large, we must have $\theta \leq \gamma$ and similarly $\theta \leq \sigma$ which gives the necessary condition:

$$
\theta \leq \min \{\gamma, \sigma\}
$$

Now if we select $\rho=\frac{1}{4}\left|x_{0}\right|$ and take $\left|x_{0}\right| \rightarrow \infty$ we get:

$$
\gamma-\theta \geq \frac{n}{p}-\frac{n}{p_{0}}
$$

and similarly with $\rho=\frac{1}{4}\left|y_{0}\right|$ and $\left|y_{0}\right| \rightarrow \infty$ :

$$
\gamma-\sigma \geq \frac{n}{p}-\frac{n}{p_{1}}
$$

By selecting $\left|x_{0}+y_{0}\right|=\left|x_{0}\right|=\left|y_{0}\right|$ and $\rho=\tau=\frac{1}{4}\left|x_{0}\right|$ and again taking $\left|x_{0}\right| \rightarrow \infty$ we get:

$$
n+\frac{n}{p}-\frac{n}{p_{0}}-\frac{n}{p_{1}} \leq \gamma+\sigma-\theta
$$

Finally, take $\left|x_{0}\right|=\left|y_{0}\right|,\left|x_{0}+y_{0}\right|=2, \tau=\frac{1}{4}\left|x_{0}\right|$ and fix $p$ to get:

$$
n-\frac{n}{p_{0}}-\frac{n}{p_{1}} \leq \gamma+\sigma
$$

We've established the following theorem:

Theorem 3.11: The inequalities (22) through (28) are necessary for the existence of 
a constant $C$ independent of $f$ and $g$ so that (19) holds.

It's not difficult to check that these necessary conditions do not quite meet sufficiency in the general case. However we will see in a later section that a special case of our weighted $L^{p}$ space convolution inequality will be of particular importance, and in that case our conditions reduce to a complete characterization up to boundary inequalities. We present that special case now. The following corollary is just the sufficient conditions on the general case with $p=p_{0}=p_{1}$.

Corollary 3.12: Let $1<r<\infty$. Then there is a constant $C$ independent of the functions $f$ and $g$ so that $\|f * g\|_{\gamma, r} \leq C\|f\|_{\alpha, r}\|g\|_{\beta, r}$ when:

i) $\alpha+\beta-\gamma>\frac{n}{r^{\prime}}$

ii) $\alpha-\gamma, \beta-\gamma, \alpha+\beta>0$

iii) $\alpha+\beta>\frac{n}{r^{\prime}}-\frac{n}{r}$

Moreover these sufficient conditions are necessary up to boundary cases, i.e. i), ii), and iii) are necessary if you replace $>$ with $\geq$.

To see that these conditions are necessary up to boundary conditions, one need only replace $p, p_{0}$, and $p_{1}$ with $r$ in the necessary conditions on the general case. In later sections we will use this subcase to help us find solutions to PDE with initial data in weighted $L^{p}$ spaces. We will see that at least in the case of the chosen weighted $L^{p}$ spaces the question of sufficiency at the boundary cases becomes irrelevant. Hence the corollary as stated is already sufficient for obtaining the best possible solutions given the techniques used. 


\section{CHAPTER 4 \\ FORMULATION OF A MILD SOLUTION}

\subsection{PARTIAL DIFFERENTIAL EQUATION FORMULATION}

In this chapter we begin our discussion of the types of partial differential equations we are interested in as well as develop the notion of a mild solution. To this end let $W$ be a Banach space with $A$ an operator on $W$ and observe the following equation:

$$
\partial_{t} u+A u=F(u, t), \quad u\left(t_{0}\right) \in W, \quad 0 \leq t_{0} \leq T
$$

We will consider these equations as evolutionary equations in $W$. Note that in this equation the partial derivative is a $W$ valued derivative with respect to $t$ and $F$ maps into $W$, so that this equation makes sense. This means that solutions to (29) will be $W$-valued functions of the real variable $t$. The solutions we will be interested in will be of a form known as a mild solution which we will develop shortly. For this we need some definitions.

\subsection{FORMULATION OF MILD SOLUTION}

Definition 4.1: Let $W$ be a Banach space and $L(W)$ the collection of bounded linear operators on $W$. We say that $T(t)$ is a semigroup of bounded linear operators on $W$ if $T(t) \in L(W) \forall t \geq 0, T(0)=I$, and $T(t) T(s)=T(t+s) \forall s, t \geq 0$.

Definition 4.2: We say that $T(t)$ is a $C_{0}$-semigroup if in addition to being a semi-group of bounded linear operators on $W$, we have continuity from the right with respect to the norm topology on $W$; i.e. that: $\lim _{t \rightarrow 0^{+}} T(t) w=w \forall w \in W$. 
Example 4.3: Let $W$ be an $L^{p}$ space with respect to Lebesgue measure. Then for $w \in W$ define $T(t) w=e^{-|x|^{2} t} w$.

Definition 4.4: Let $T(t)$ be a $C_{0}$-semigroup on $W$. The infinitesimal generator of $T(t)$ is an operator $A$ on $W$ with domain D satisfying:

$$
\begin{gathered}
D(A)=\left\{w \in W: \lim _{h \rightarrow 0^{+}} \frac{T(h)-I}{h} w \in W\right\} \\
A w=\left.\lim _{h \rightarrow 0^{+}} \frac{d^{+}(T(t+h) w)}{d t}\right|_{t=0}
\end{gathered}
$$

In other words $A w$ is the righthand $W$ valued derivative of $T(t) w$ evaluated at $t=0$.

Example 4.5: If $W$ is an $L^{p}$ space with respect to Lebesgue measure and $A w=|x|^{2} w$ then $-A$ generates $e^{-|x|^{2} t}$. This motivates the notation $T(t)=e^{-A t}$ when $-A$ is the infinitesimal generator of $T(t)$. We will return to infinitesimal generators of this form in later sections.

We are now prepared to formally state the definition of a mild solution to the initial value problem.

Definition 4.6: (Mild Solution) Let $-A$ be the infinitesimal generator of a $C_{0^{-}}$ semigroup $e^{-A t}$ on $W$. We say that $u$ is a mild solution in $W$ on the interval $\left[t_{0}, T\right] \subset \mathbb{R}^{+}$of the initial value problem (29) if $u:\left[t_{0}, T\right] \rightarrow W$ is continuous with respect to the norm topology on $W$ and a solution of the integral equation:

$$
u(t)=e^{-A\left(t-t_{0}\right)} u_{0}+\int_{t_{0}}^{t} e^{-A(t-s)} F(u(s), s) d s, \quad t_{0} \leq t \leq T
$$


Henceforth we will assume $t_{0}=0$. It's important to note that the integral is a $W$ valued integral in the Bochner sense so that all terms in the above equation are $W$ valued functions of $t$. Our methods will involve demonstrating that under certain conditions, the integral equation above is a contraction mapping of the form $S u=g+b(u, u)$ to which we can apply the Banach fixed point theorem to obtain solutions. To this end we state the following existence theorem.

\subsection{THE EXISTENCE THEOREM}

Theorem 4.7: (Existence Theorem) Let $\Sigma$ be a Banach space with norm $\|\cdot\|_{\Sigma}$ and let $q: \Sigma \rightarrow[0, \infty)$ be a subaddtive functional satisfying $q \leq\|\cdot\|_{\Sigma}$. Suppose $g \in \Sigma$ and $b: \Sigma \times \Sigma \rightarrow \Sigma$ is bilinear. Let: $E=\{u \in \Sigma \mid q(u-g) \leq q(g)\}$ If there exists $0<\theta<\frac{1}{2}$ such that:

$$
\|b(u, v)\|_{\Sigma} \leq \theta q(v)
$$

whenever $u \in E$ and $v \in \Sigma$, and

$$
\|b(u, v)\|_{\Sigma} \leq \theta q(u)
$$

whenever $u \in \Sigma$ and $v \in E$ then there is a unique $u \in \Sigma$ satisfying $u=g+b(u, u)$.

See [12] for a proof. 


\section{CHAPTER 5}

\section{FINDING A MILD SOLUTION TO A SIMPLE PDE}

\subsection{PRESENTING A SIMPLE PDE}

In this chapter we will use a simple initial value problem in one spatial dimension to demonstrate our methods for finding mild solutions to partial differential equations. The techniques follow the methods used by Animikh Biswas and this dissertation author's advisor, David Swanson in their analysis of the Kuramoto-Sivashinsky equation [12]. The methods used here differ in that we will employ our own improved convolution inequalities over the simple convolution inequalities used in their original analysis. We will look more closely at the KSE in the next chapter. Consider the following:

$$
\frac{\partial}{\partial t} u=\frac{\partial^{2}}{\partial x^{2}} u+\frac{1}{2} \frac{\partial}{\partial x} u^{2}+u^{2} \quad u_{0}=u(x, 0)
$$

where solutions are of the form $u(x, t)$. These can be interpreted as mappings that assign to each value of $t$ a function of a single real variable $x$. We will look for mild solutions in Fourier space with initial data in $W=L_{\alpha}^{p}(\mathbb{R})$. The membership of the Fourier transform of $u_{0}$ in $L_{\alpha}^{p}$ is related to the smoothness of $u_{0}$. In particular if $u$ is a tempered distribution then $\hat{u} \in L_{\alpha}^{2}(\mathbb{R})$ if and only if $u$ is in the Sobolev space $H^{\alpha}$. A lower value of $\alpha$ corresponds to fewer orders of differentiability and hence allows for rougher initial data. We proceed by applying the Fourier transform.

$$
\begin{array}{r}
\frac{\partial}{\partial t} \hat{u}=-x^{2} \hat{u}+\hat{u} * \hat{u}_{x}+\hat{u} * \hat{u} \\
\frac{\partial}{\partial t} \hat{u}=-x^{2} \hat{u}+\int_{\mathbb{R}} \hat{u}_{x}(z) \hat{u}(x-z) d z+\int_{\mathbb{R}} \hat{u}(z) \hat{u}(x-z) d z \\
\frac{\partial}{\partial t} \hat{u}=-x^{2} \hat{u}+\int_{\mathbb{R}}(1+i z) \hat{u}(z) \hat{u}(x-z) d z
\end{array}
$$


which suggests the definition of the operator $A$ and the bilinear operator $B$ so that we can write this as $u_{t}=-A u+B(u, u)$ after omitting the hats for convenience. After converting to integral form this is in the form of the equation satisfied by the mild solutions.:

$$
u(t)=e^{-t A} u_{0}-\int_{0}^{t} e^{-(t-s) A} B(u, u)(s) d s
$$

Definition 5.1: Let $\phi(x)$ be a nonnegative function satisfying $\phi(x+y) \leq \phi(x)+$ $\phi(y)+C$. We say that a solution $u$ of (30) on the time interval $0 \leq t \leq T$ is Gevrey regular if:

$$
\sup _{0 \leq t \leq T}\|u(t)\|_{t \phi, \alpha, p}<\infty
$$

where the generalized Gevrey norm used above is defined with respect to the function $\phi$ in $n$ dimensions by:

$$
\|u(t)\|_{\phi, w, p}=\left(\int_{\mathbb{R}^{n}} e^{p \phi(x)}|u(t)|^{p} w(x) d x\right)^{\frac{1}{p}}
$$

Take note of the fact that these norms are computed with respect to the space variable $x$ and not the time variable so that $\|u(t)\|_{t \phi, \alpha, p}$ still depends on $t$. Gevrey regularity guarantees that the solutions decrease rapidly in time so as to counteract the growing exponential factor. It's particularly useful for obtaining estimates on radii of analyticity of solutions in terms of the initial data. We now state and prove the existence of a Gevrey regular solution to (30).

\subsection{SOLVING A SIMPLE PDE}

Theorem 5.2: (Existence of a Mild Solution) Let $\alpha>\frac{1}{p^{\prime}}$ and $u_{0} \in L_{p}^{\alpha}(\mathbb{R})$ with $1<p<\infty$. There exists $T>0$ and a corresponding $u \in C\left([0, T], L_{p}^{\alpha}(\mathbb{R})\right)$ with $u(0)=u_{0}$ satisfying (30). Moreover $u$ is Gevrey regular. 
In the propositions and theorems that follow assume that $C$ is always independent of the functions $u$ and $v$ and the variable $T$ after it is introduced. Occasionally this will be restated for emphasis along with any other dependencies or lack thereof that are important.

Theorem 5.3. There exists $C \geq 0$ such that:

$$
\|B(u, v)\|_{\phi, \sigma, p} \leq C\|u\|_{\phi, \gamma+1, p}\|v\|_{\phi, \gamma+1, p}
$$

so long as:

$$
1<p<\infty \quad 2 \gamma-\sigma>\frac{1}{p^{\prime}}, \quad \gamma>\sigma, \quad \gamma>0, \quad 2 \gamma>\frac{1}{p^{\prime}}-\frac{1}{p}
$$

Proof. The stated conditions are a special case of our own convolution inequality. Thus there exists $C \geq 0$ such that:

$$
\|u * v\|_{\sigma, p} \leq C\|u\|_{\gamma, p}\|v\|_{\gamma, p}
$$

Note that $\|B(u, v)\|_{\sigma, p}=\|u *(1+i x) v\|_{\sigma, p}$. Apply the convolution inequality and use the fact that the norm is nondecreasing in $\gamma$ and $|1+i x|^{p} \leq(1+|x|)^{p}$ to obtain:

$$
\|B(u, v)\|_{\sigma, p} \leq C\|u\|_{\gamma+1, p}\|v\|_{\gamma+1, p}
$$

Finally, we extend to the Gevrey norm using an identical argument to the one in [12].

Lemma 5.4: $\forall \eta>0$ and $\theta \in \mathbb{R}$ we have:

$$
\sup _{x \in \mathbb{R}} e^{-\eta x^{2}}(1+|x|)^{\theta} \leq C_{\theta}\left(1+\eta^{-\frac{\theta}{2}}\right)
$$


Proof. It's clear when $\theta \leq 0$ because the lefthand side is bounded by 1 . Otherwise note that $e^{-\eta x^{2}}(1+|x|)^{\theta} \leq C_{\theta} e^{-\eta x^{2}}\left(1+|x|^{\theta}\right) \leq C_{\theta}\left(1+e^{-\eta x^{2}}|x|^{\theta}\right)$. Now apply the first derivative test to the final term.

Theorem 5.5. Under the same conditions as Theorem 5.3 we have:

$$
\left\|e^{-\eta A} B(u, v)\right\|_{\phi, \delta, p} \leq C\left(1+\eta^{-\frac{\delta}{2}+\frac{\sigma}{2}}\right)\|u\|_{\phi, \gamma+1, p}\|v\|_{\phi, \gamma+1, p} \quad \forall \eta>0 \quad \forall \delta \in \mathbb{R}
$$

with the constant independent of $\eta$.

Proof.

$$
e^{-p \eta x^{2}}(1+|x|)^{p \delta}=e^{-p \eta x^{2}}(1+|x|)^{p \delta-\sigma p}(1+|x|)^{\sigma p} \leq C_{\delta, \sigma, p}\left(1+\eta^{-\frac{p \delta}{2}+\frac{\sigma p}{2}}\right)(1+|x|)^{\sigma p}
$$

where the last inequality follows from the previous estimate. Now:

$$
\begin{array}{r}
\left\|e^{-\eta A} B(u, v)\right\|_{\phi, \delta, p}=\left(\int_{\mathbb{R}} e^{p \phi} e^{-p \eta x^{2}}(1+|x|)^{\delta p}|B(u, v)|^{p} d x\right)^{\frac{1}{p}} \\
\leq C\left(1+\eta^{-\frac{\delta}{2}+\frac{\sigma}{2}}\right)\left(\int_{\mathbb{R}} e^{p \phi}(1+|x|)^{\sigma p}|B(u, v)|^{p} d x\right)^{\frac{1}{p}} \\
=C\left(1+\eta^{-\frac{\delta}{2}+\frac{\sigma}{2}}\right)\|B(u, v)\|_{\phi, \sigma, p} \\
\leq C\left(1+\eta^{-\frac{\delta}{2}+\frac{\sigma}{2}}\right)\|u\|_{\phi, \gamma+1, p}\|v\|_{\phi, \gamma+1, p}
\end{array}
$$

Theorem 5.6 $\forall 0 \leq s \leq t \leq \infty$ and $\forall \alpha \in \mathbb{R}$ we have:

$$
\left\|e^{-(t-s) A} u\right\|_{t \phi, \alpha, p} \leq e^{C(t-s)}\left\|e^{-\frac{(t-s)}{2} A} u\right\|_{s \phi, \alpha, p}
$$

Proof. Define $C=\frac{1}{2} \sup _{x \in \mathbb{R}}\left(2 \phi(x)-|x|^{2}\right)$. Since $\phi$ is subadditive up to a constant, it has sublinear growth and so the squared term guarantees $C<\infty$. Some rearranging 
guarantees that:

$$
|x|^{2}-\phi(x) \geq \frac{|x|^{2}}{2}-C
$$

And so:

$$
e^{-p(t-s)\left(|x|^{2}-\phi(x)\right)} \leq e^{-p(t-s)\left(\frac{|x|^{2}}{2}-C\right)}=e^{-p \frac{(t-s)}{2}|x|^{2}} e^{p C(t-s)}
$$

Therefore:

$$
\begin{array}{r}
\left\|e^{-(t-s) A} u\right\|_{t \phi, \alpha, p}^{p}=\int_{\mathbb{R}} e^{-p(t-s)|x|^{2}} e^{p t \phi(x)}(1+|x|)^{\alpha p}|u(x)|^{p} d x \\
=\int_{\mathbb{R}} e^{p s \phi(x)} e^{-p(t-s)|x|^{2}+p t \phi(x)-p s \phi(x)}(1+|x|)^{\alpha p}|u(x)|^{p} d x \\
=\int_{\mathbb{R}} e^{p s \phi(x)} e^{-p(t-s)|x|^{2}+p(t-s) \phi(x)}(1+|x|)^{\alpha p}|u(x)|^{p} d x \\
=\int_{\mathbb{R}} e^{p s \phi(x)} e^{-p(t-s)\left(|x|^{2}-\phi(x)\right)}(1+|x|)^{\alpha p}|u(x)|^{p} d x \\
\leq \int_{\mathbb{R}} e^{p s \phi(x)} e^{-p(t-s)\left(\frac{|x|^{2}}{2}-C\right)}(1+|x|)^{\alpha p}|u(x)|^{p} d x \\
=e^{p C(t-s)} \int_{\mathbb{R}} e^{p s \phi(x)} e^{-p \frac{(t-s)}{2}|x|^{2}}(1+|x|)^{\alpha p}|u(x)|^{p} d x \\
=e^{p C(t-s)}\left\|e^{-\frac{(t-s)}{2}} A u\right\|_{s \phi, \alpha, p}^{p}
\end{array}
$$

Theorem 5.7. $\forall t \geq 0, \alpha, \beta \in \mathbb{R}$ we have:

$$
\left\|e^{-t A} u\right\|_{t \phi, \alpha+\beta, p} \leq e^{C t} C\left(1+t^{-\frac{\beta}{2}}\right)\|u\|_{\alpha, p}
$$

where the constants do not depend on $t$.

Proof. First we apply Theorem 5.6 with $s=0$ :

$$
\left\|e^{-t A} u\right\|_{t \phi, \alpha+\beta, p} \leq e^{C t}\left\|e^{-\frac{t}{2} A} u\right\|_{\alpha+\beta, p}
$$


Now observe that:

$$
\begin{gathered}
e^{-\frac{p t}{2}|x|^{2}}(1+|x|)^{(\alpha+\beta) p}=e^{-\frac{p t}{2}|x|^{2}}(1+|x|)^{\beta p}(1+|x|)^{\alpha p} \\
\leq C\left(1+\frac{p t^{-\frac{\beta p}{2}}}{2}\right)(1+|x|)^{\alpha p} \\
=C\left(1+t^{-\frac{\beta p}{2}}\right)(1+|x|)^{\alpha p}
\end{gathered}
$$

Therefore:

$$
\begin{gathered}
\left\|e^{-\frac{t}{2} A} u\right\|_{\alpha+\beta, p}=\left(\int_{\mathbb{R}} e^{-\frac{p t}{2}|x|^{2}}(1+|x|)^{(\alpha+\beta) p}|u(x)|^{p} d x\right)^{\frac{1}{p}} \\
\leq C\left(1+t^{-\frac{\beta}{2}}\right)\left(\int_{\mathbb{R}}(1+|x|)^{\alpha p}|u(x)|^{p} d x\right)^{\frac{1}{p}} \\
=C\left(1+t^{-\frac{\beta}{2}}\right)\|u\|_{\alpha, p}
\end{gathered}
$$

In order to prove the main theorem of this section we will construct an appropriate Banach space and associated components of the existence theorem, and then use the above estimates to show that the hypotheses of the existence theorem are satisfied.

Proof. (Theorem 5.2) Let $\alpha>\frac{1}{p^{\prime}}$ and $u_{0} \in L_{\alpha}^{p}(\mathbb{R})$. Select $\beta>0$ and $\sigma \in \mathbb{R}$ so that the following holds:

$$
\begin{gathered}
2(\alpha+\beta-1)-\sigma>\frac{1}{p^{\prime}}, \quad \alpha+\beta-1>\sigma, \quad \alpha+\beta-1>0, \quad 2(\alpha+\beta-1)>\frac{1}{p^{\prime}}-\frac{1}{p} \\
\beta<1, \quad \frac{\alpha}{2}-\frac{\sigma}{2}<1, \quad \frac{\alpha+\beta}{2}-\frac{\sigma}{2}<1, \quad \frac{\sigma}{2}-\frac{\alpha}{2}+1-\beta>0
\end{gathered}
$$

Let's convince ourselves that this is possible and that the requirement on $\alpha$ can't be relaxed. We will begin by eliminating $\sigma$. Rearranging the conditions shows that 
ultimately $\sigma$ must satisfy:

$$
\max \{\alpha-2, \quad \alpha+\beta-2, \quad \alpha-2+2 \beta\}<\sigma<\min \left\{2(\alpha+\beta-1)-\frac{1}{p^{\prime}}, \quad \alpha+\beta-1\right\}
$$

which means that choosing a valid $\sigma$ requires choosing $B>0$ so that:

$$
\begin{gathered}
\alpha-2+2 \beta<\min \left\{2 \alpha+2 \beta-2-\frac{1}{p^{\prime}}, \quad \alpha+\beta-1\right\} \\
\alpha+\beta-1>0, \quad \alpha+\beta-1>\frac{1}{2 p^{\prime}}-\frac{1}{2 p}, \quad \beta<1
\end{gathered}
$$

The first line combined with $0<\beta<1$ reduces to $\alpha>\frac{1}{p^{\prime}}$. Now we can rearrange and eliminate $\beta$. The second line gives the following conditions:

$$
\max \left\{0, \quad 1-\alpha, \quad \frac{1}{2 p^{\prime}}-\frac{1}{2 p}-\alpha+1\right\}<\beta<1
$$

but this interval of validity for $\beta$ is already guaranteed nonempty due to the requirement that $\alpha>\frac{1}{p^{\prime}}$. Define $g(t)=e^{-A t} u_{0}$. We claim that:

$$
\begin{gathered}
\sup _{0 \leq t \leq T}\|g(t)\|_{t \phi, \alpha, p} \leq e^{C T}\left\|u_{0}\right\|_{\alpha, p}<\infty \\
\sup _{0 \leq t \leq T} t^{\frac{\beta}{2}}\|g(t)\|_{t \phi, \alpha+\beta, p} \leq C e^{C T}\left(T^{\frac{\beta}{2}}+1\right)\left\|u_{0}\right\|_{\alpha, p}<\infty
\end{gathered}
$$

To get (31) apply Theorem 5.6 with $s=0$ and use the fact that $\sup _{0 \leq t \leq T} e^{-\frac{t}{2}|x|^{2}}=1$ :

$$
\|g(t)\|_{t \phi, \alpha, p}=\left\|e^{-t A} u_{0}\right\|_{t \phi, \alpha, p} \leq e^{C t}\left\|e^{-\frac{t}{2} A} u_{0}\right\|_{\alpha, p} \leq e^{C T}\left\|u_{0}\right\|_{\alpha, p}
$$

To get (32) we again apply Theorem 5.6 with $s=0$ :

$$
\|g(t)\|_{t \phi, \alpha+\beta, p}=\left\|e^{-t A} u_{0}\right\|_{t \phi, \alpha+\beta, p} \leq e^{C t}\left\|e^{-\frac{t}{2} A} u_{0}\right\|_{\alpha+\beta, p}
$$


Then we use the fact that on the given interval, $1 \leq e^{\frac{t}{2} \phi(x)}$ and then Theorem 5.7 to get:

$$
\left\|e^{-\frac{t}{2} A} u_{0}\right\|_{\alpha+\beta, p} \leq\left\|e^{-\frac{t}{2} A} u_{0}\right\|_{\frac{t}{2} \phi . \alpha+\beta, p} \leq C e^{C t}\left(1+t^{-\frac{\beta}{2}}\right)\left\|u_{0}\right\|_{\alpha, p}
$$

Now just multiply by $t^{\frac{\beta}{2}}$ on both sides. Define:

$$
\begin{gathered}
\|u\|_{\Sigma^{\prime}}=\sup _{0 \leq t \leq T} t^{\frac{\beta}{2}}\|u\|_{t \phi, \alpha+\beta, p} \\
\|u\|_{\Sigma}=\max \left\{\sup _{0 \leq t \leq T}\|u\|_{t \phi, \alpha, p}, \sup _{0 \leq t \leq T} t^{\frac{\beta}{2}}\|u\|_{t \phi, \alpha+\beta, p}\right\}
\end{gathered}
$$

And consequently define the Banach space:

$$
\Sigma=\left\{u \in C\left([0, T], L_{p}^{\alpha}(\mathbb{R})\right) \mid\|u\|_{\Sigma}<\infty\right\}
$$

(31) and (32) guarantee that $g \in \Sigma$ and that:

$$
\|g\|_{\Sigma^{\prime}} \leq C e^{C T}\left(T^{\frac{\beta}{2}}+1\right)\left\|u_{0}\right\|_{\alpha, p}
$$

Define:

$$
b(u, v)=-\int_{0}^{t} e^{-(t-s) A} B(u, v)(s) d s
$$

We claim that if $u, v \in \Sigma$ then $b(u, v) \in \Sigma$ and:

$$
\|b(u, v)\|_{\Sigma} \leq C e^{C T}\left(T^{1-\frac{\beta}{2}}+T^{1-\beta}+T^{\frac{\sigma}{2}-\frac{\alpha}{2}+1-\beta}\right)\|u\|_{\Sigma^{\prime}}\|v\|_{\Sigma^{\prime}}
$$

To see this let $\delta \in \mathbb{R}, 0<s<t<T$, and write $\gamma=\alpha+\beta-1$. Then by Theorem 5.5 we have:

$$
\left\|e^{-\frac{1}{2}(t-s) A} B(u, v)(s)\right\|_{s \phi, \delta, p} \leq C\left(1+(t-s)^{-\frac{\delta}{2}+\frac{\sigma}{2}}\right)\|u\|_{s \phi, \gamma+1, p}\|v\|_{s \phi, \gamma+1, p}
$$




$$
\leq C\left(1+(t-s)^{-\frac{\delta}{2}+\frac{\sigma}{2}}\right) s^{-\beta}\|u\|_{\Sigma^{\prime}}\|v\|_{\Sigma^{\prime}}
$$

Now we use the triangle inequality, Theorem 5.6, and then the previous inequality:

$$
\begin{array}{r}
\|b(u, v)\|_{t \phi, \delta, p} \leq \int_{0}^{t}\left\|e^{-(t-s) A} B(u, v)\right\|_{t \phi, \delta, p} d s \\
\leq e^{C T} \int_{0}^{t}\left\|e^{-\frac{(t-s)}{2} A} B(u, v)\right\|_{s \phi, \delta, p} d s \\
\leq C e^{C T}\|u\|_{\Sigma^{\prime}}\|v\|_{\Sigma^{\prime}} \int_{0}^{t}\left(1+(t-s)^{-\frac{\delta}{2}+\frac{\sigma}{2}}\right) s^{-\beta} d s
\end{array}
$$

This integral converges when $\beta<1$ and $\frac{\delta}{2}-\frac{\sigma}{2}<1$ and is then bounded by a constant times $\left(t^{1-\beta}+t^{\frac{\sigma}{2}-\frac{\delta}{2}-\beta+1}\right)$. (To see this substitute $s=r t$.) The hypotheses guarantee that these two conditions are met with $\delta=\alpha$ and $\delta=\alpha+\beta$. Therefore we get the following estimates:

$$
\|b(u, v)\|_{t \phi, \alpha, p} \leq C e^{C T}\left(t^{1-\beta}+t^{\frac{\sigma}{2}-\frac{\alpha}{2}-\beta+1}\right)\|u\|_{\Sigma^{\prime}}\|v\|_{\Sigma^{\prime}}
$$

and

$$
t^{\frac{\beta}{2}}\|b(u, v)\|_{t \phi, \alpha+\beta, p} \leq C e^{C T}\left(t^{1-\frac{\beta}{2}}+t^{\frac{\sigma}{2}-\frac{\alpha}{2}+1-\beta}\right)\|u\|_{\Sigma^{\prime}}\|v\|_{\Sigma^{\prime}}
$$

Since the conditions on $\alpha, \beta$, and $\sigma$ guarantee that the exponents on $t$ are positive, we can combine these two estimates to get (34). Define $E=\left\{u \in \Sigma \mid\|u-g\|_{\Sigma^{\prime}} \leq\|g\|_{\Sigma^{\prime}}\right\}$ Now suppose $u \in E$ and $v \in \Sigma$. Then:

$$
\|u\|_{\Sigma^{\prime}} \leq\|u-g\|_{\Sigma^{\prime}}+\|g\|_{\Sigma^{\prime}} \leq 2\|g\|_{\Sigma^{\prime}}
$$

So that from (34) and then (33) we get:

$$
\begin{aligned}
& \|b(u, v)\|_{\Sigma} \leq C e^{C T}\left(T^{1-\frac{\beta}{2}}+T^{1-\beta}+T^{\frac{\sigma}{2}-\frac{\alpha}{2}+1-\beta}\right)\|g\|_{\Sigma^{\prime}}\|v\|_{\Sigma^{\prime}} \\
& \leq C e^{C T}\left(T^{\frac{\beta}{2}}+1\right)\left(T^{1-\frac{\beta}{2}}+T^{1-\beta}+T^{\frac{\sigma}{2}-\frac{\alpha}{2}+1-\beta}\right)\left\|u_{0}\right\|_{\alpha, p}\|v\|_{\Sigma^{\prime}}
\end{aligned}
$$


And likewise when $u \in \Sigma$ and $v \in E$ we get:

$$
\|b(u, v)\|_{\Sigma} \leq C e^{C T}\left(T^{\frac{\beta}{2}}+1\right)\left(T^{1-\frac{\beta}{2}}+T^{1-\beta}+T^{\frac{\sigma}{2}-\frac{\alpha}{2}+1-\beta}\right)\left\|u_{0}\right\|_{\alpha, p}\|u\|_{\Sigma^{\prime}}
$$

Since $C$ has no $T$ dependence we can just choose $\mathrm{T}$ small enough so that:

$$
\theta=C e^{C T}\left(T^{\frac{\beta}{2}}+1\right)\left(T^{1-\frac{\beta}{2}}+T^{1-\beta}+T^{\frac{\sigma}{2}-\frac{\alpha}{2}+1-\beta}\right)\left\|u_{0}\right\|_{\alpha, p}<\frac{1}{2}
$$

Now apply Theorem 4.7 with $q(\cdot)=\|\cdot\|_{\Sigma^{\prime}}$ to obtain $u \in \Sigma$ satisfying $u=g+b(u, u)$ which is precisely a solution to (30) as desired. 
CHAPTER 6

IMPROVED SOLUTIUONS TO THE KURAMOTO-SIVASHINSKY EQUATION

\subsection{THE KURAMOTO-SIVASHINSKY EQUATION}

We turn our attention to a more established PDE in the literature. In this section

we will apply the techniques developed in the the previous section to the KuramotoSivashinsky equation (KSE). This section will serve as an improvement to the results obtained by David Swanson and Animikh Biswas in their analysis of the KSE [12]. The KSE is:

$$
u_{t}+\Delta^{2} u+\Delta u+\frac{1}{2}|\nabla u|^{2}=0 \quad u(x, 0)=u_{0}(x)
$$

with $u(x, t)$ defined on $\mathbb{R}^{n} \times[0, T]$.

\subsection{THE RESULT TO BE IMPROVED}

In their paper, Swanson and Biswas found conditions on the parameter $\alpha$ under which the KSE has Gevrey regular mild solutions in Fourier Space with initial data in $L_{\alpha}^{p}\left(\mathbb{R}^{n}\right)$ just as was done with the one dimensional PDE in the previous section. Using convolution inequalities obtained via simple integral estimates, the sufficient conditions for existence were that $\max \left\{\frac{n}{2 p^{\prime}}-1, \frac{n}{p^{\prime}}-2\right\}<\alpha<\frac{n}{p^{\prime}}+1$. In this section we will employ our improved convolution inequalities obtained via interpolation to completely remove the upper bound on $\alpha$ and decrease the lower bound. To this end we apply the Fourier transform to (35) as was done in the previous section. Then we use properties of the Fourier transform and omit the hats for convenience and get:

$$
\frac{\partial}{\partial t} u(x, t)+|x|^{4} u(x, t)-|x|^{2} u(x, t)-\frac{1}{2} \int_{\mathbb{R}^{n}} z \cdot(x-z) u(z) u(x-z) d z=0
$$


We define the operator $A$ by: $A u(x, t)=\left(|x|^{4}-|x|^{2}\right) u(x, t)$ and $B(u, v)$ defined by:

$$
B(u, v)=-\frac{1}{2} \int_{\mathbb{R}^{n}} z \cdot(x-z) u(z) u(x-z) d z
$$

so that (36) can be expressed as:

$$
\frac{\partial}{\partial t} u(x, t)+\left(A^{2}-A\right) u(x, t)+B(u, u)(x, t)=0
$$

so that integral form for the formulation of the mild solution with initial condition $u_{0}=u(0)$ is:

$$
u(t)=e^{-t\left(A^{2}-A\right)} u_{0}-\int_{0}^{t} e^{-(t-s)\left(A^{2}-A\right)} B(u, u)(s) d s
$$

We now state our main result for this section:

Theorem 6.1: (Existence of a Mild Solution) Let $\alpha>\max \left\{-1, \frac{n}{p^{\prime}}-2, \frac{n}{2 p^{\prime}}-\frac{n}{2 p}-1\right\}$ and $u_{0} \in L_{\alpha}^{p}\left(\mathbb{R}^{n}\right)$ with $1<p<\infty$. There exists $T>0$ and a corresponding $u \in C\left([0, T], L_{p}^{\alpha}(\mathbb{R})\right)$ with $u(0)=u_{0}$ satisfying $(37)$. Moreover $u$ is Gevrey regular.

The proofs for virtually all of the propositions that follow are nearly identical to the previous section with a few minor changes. Therefore many proofs and details will be omitted in this section. For the rest of this section assume that $\phi$ denotes a non-negative sub-additive function up to a constant; i.e. that there exists $C$ such that for all $x, y \in \mathbb{R}^{n}$, we have that $\phi(x+y) \leq \phi(x)+\phi(y)+C$. Also in the propositions and theorems that follow assume that $C$ is always independent of the functions $u$ and $v$ and the variable $T$ after it is introduced. Occasionally this will be restated for emphasis along with any other dependencies or lack thereof that are important. 
Proposition 6.2: Suppose that $1<p<\infty$. There exists $C$ independent of $\eta, u$ and $v$ such that:

$$
\left\|e^{-\eta\left(A^{2}-A\right)} B(u, v)\right\|_{\phi, \delta, p} \leq C e^{\frac{\eta}{2}}\left(1+\eta^{-\frac{\delta}{4}+\frac{\sigma}{4}}\right)\|u\|_{\phi, \gamma+1, p}\|v\|_{\phi, \gamma+1, p}, \quad \forall \delta \in \mathbb{R}, \eta>0
$$

whenever the following conditions are satisfied:

$$
2 \gamma-\sigma>\frac{n}{p^{\prime}}, \quad \gamma>\sigma, \quad \gamma>0, \quad 2 \gamma>\frac{n}{p^{\prime}}-\frac{n}{p}
$$

Similarly to the previous section, (38) was obtained by using convolution inequalities. Notice that again, the conditions are exactly a sub-case of the corollary of our own weighted $L^{p}$ space convolution inequality with the appropriate indices replaced. Proposition 6.2 is analogous to Lemma 16 in [12]. Note the more general conditions obtained with our improved convolution inequalities. Our improvement of their results hinges on this proposition.

Proposition 6.3: $\forall 0 \leq s \leq t \leq \infty$ and $\forall \alpha \in \mathbb{R}$ we have:

$$
\left\|e^{-(t-s)\left(A^{2}-A\right)} u\right\|_{t \phi, \alpha, p} \leq e^{C(t-s)}\left\|e^{-\frac{(t-s)}{2}\left(A^{2}-A\right)} u\right\|_{s \phi, \alpha, p}
$$

with the constant dependent upon at most $\phi$.

Proposition 6.4: $\forall t \geq 0, \alpha, \beta \in \mathbb{R}$ we have:

$$
\left\|e^{-t\left(A^{2}-A\right)} u\right\|_{t \phi, \alpha+\beta, p} \leq e^{C t} C\left(1+t^{-\frac{\beta}{4}}\right)\|u\|_{\alpha, p}
$$

where the constants do not depend on $t$. 


\subsection{SOLVING THE KURAMOTO-SIVASHINSKY EQUATION}

Proof of Theorem 6.1. Suppose that $\alpha>\max \left\{-1, \frac{n}{p^{\prime}}-2, \frac{n}{2 p^{\prime}}-\frac{n}{2 p}-1\right\}$ and $u_{0} \in L_{\alpha}^{p}\left(\mathbb{R}^{n}\right)$ with $1<p<\infty$. By arguing as in the previous session it can be shown these conditions on $\alpha$ are necessary and sufficient for selecting $\beta$ and $\sigma$ so that the following is satisfied:

$$
\begin{gathered}
2(\alpha+\beta-1)-\sigma>\frac{n}{p^{\prime}}, \quad \alpha+\beta-1>\sigma, \quad \alpha+\beta-1>0, \quad 2(\alpha+\beta-1)>\frac{n}{p^{\prime}}-\frac{n}{p} \\
0<\beta<2, \quad \frac{\alpha+\beta}{4}-\frac{\sigma}{4}<1, \quad \frac{\alpha}{4}-\frac{\sigma}{4}<1, \quad \frac{\sigma}{4}-\frac{\alpha}{4}+1-\frac{\beta}{2}>0
\end{gathered}
$$

Like in the previous chapter, the first line of inequalities guarantees we will have Proposition 6.2 at our disposal with $\gamma=\alpha+\beta-1$ and the second line guarantees that the appropriate integrals converge and that we have the necessary control in selecting $\theta<\frac{1}{2}$ for the existence theorem. To this end let $T>0$ be arbitrary and define $g(t)=e^{-\left(A^{2}-A\right) t} u_{0}$. Then define $\Sigma$ and $\Sigma^{\prime}$ like so:

$$
\begin{gathered}
\|u\|_{\Sigma^{\prime}}=\sup _{0 \leq t \leq T} t^{\frac{\beta}{4}}\|u\|_{t \phi, \alpha+\beta, p} \\
\|u\|_{\Sigma}=\max \left\{\sup _{0 \leq t \leq T}\|u\|_{t \phi, \alpha, p}, \sup _{0 \leq t \leq T} t^{\frac{\beta}{4}}\|u\|_{t \phi, \alpha+\beta, p}\right\}
\end{gathered}
$$

And then the Banach space:

$$
\Sigma=\left\{u \in C\left([0, T], L_{p}^{\alpha}(\mathbb{R})\right) \mid\|u\|_{\Sigma}<\infty\right\}
$$

Note that there are some slight changes in these norms as compared to the previous section. Propositions 6.3 and 6.4 can be used to show that $g \in \Sigma$ and there exists $C$ 
and $C^{\prime}$ independent of $T$ so that:

$$
\|g\|_{\Sigma^{\prime}} \leq C e^{C^{\prime} T}\left(T^{\frac{\beta}{4}}+1\right)\left\|u_{0}\right\|_{\alpha, p}
$$

Now for $0 \leq t \leq T$ define:

$$
b(u, v)=-\int_{0}^{t} e^{-(t-s)\left(A^{2}-A\right)} B(u, v)(s) d s
$$

By repeating the procedure of the previous section, the second line of the conditions (39) can be used along with Propositions 6.2 and 6.3 to obtain the following proposition:

Proposition 6.5: If $u, v \in \Sigma$ then $b(u, v) \in \Sigma$ and there are constants $C$ and $C^{\prime}$ independent of $T$ such that:

$$
\|b(u, v)\|_{\Sigma} \leq C e^{C^{\prime} T}\left(T^{1-\frac{\beta}{4}}+T^{1-\frac{\beta}{2}}+T^{\frac{\sigma}{4}-\frac{\alpha}{4}+1-\frac{\beta}{2}}\right)\|u\|_{\Sigma^{\prime}}\|v\|_{\Sigma^{\prime}}
$$

Once again we define $E=\left\{u \in \Sigma \mid\|u-g\|_{\Sigma^{\prime}} \leq\|g\|_{\Sigma^{\prime}}\right\}$ and use Proposition 6.5 to obtain $\theta$ needed for the existence theorem:

$$
\theta=C e^{C^{\prime} T}\left(T^{\frac{\beta}{4}}+1\right)\left(T^{1-\frac{\beta}{4}}+T^{1-\frac{\beta}{2}}+T^{\frac{\sigma}{4}-\frac{\alpha}{4}+1-\frac{\beta}{2}}\right)\left\|u_{0}\right\|_{\alpha, p}
$$

which due to the exponents on $T$ being positive, can be made less than $\frac{1}{2}$ by selecting $T>0$ small enough. Applying the Theorem 4.7 gives $u \in \Sigma$ satisfying (37), which is a Gevrey regular mild solution to the KSE as desired. 


\section{CHAPTER 7 \\ CONVOLUTION INEQUALITIES IN WEIGHTED LORENTZ SPACES}

\subsection{WHY LORENTZ SPACES?}

In this chapter we define and derive convolution inequalities involving weighted Lorentz spaces in hopes that we may investigate solutions to partial differential equations with initial data in Lorentz spaces. Lorentz spaces are generalizations of the usual $L_{p}$ spaces and much of this author's understanding of how they work is due to the masters thesis of Erik Kristiansson [6]. The convolution inequalities obtained in this chapter for Lorentz spaces with our weights of the form $w(x)=(1+|x|)^{\alpha p}$ were studied by Kerman [7] for weights of the form $w(x)=|x|^{\alpha p}$. We will employ very similar methods here; i.e. we will derive convolution inequalities of the form seen previously in this dissertation, again using techniques from interpolation. There are numerous ways to define the Lorentz spaces but here we will present the definition used by Kerman.

\subsection{DEFINITION AND FACTS OF LORENTZ SPACES}

Definition 7.1:(Weighted Lorentz Spaces) We define the weighted Lorentz or $L_{p, q, w}$ spaces to be the set of Lebesgue measurable functions on $\mathbb{R}^{n}$ for which the following is finite:

$$
\begin{array}{r}
\|f\|_{p, q ; w}=\left(q \int_{0}^{\infty} s^{q-1} \mu_{f}(s)^{\frac{q}{p}} d s\right)^{\frac{1}{q}}, \quad 1<p<\infty, 1 \leq q<\infty \\
\sup _{s>0} s \mu_{f}(s)^{\frac{1}{p}}, \quad 1<p<\infty, q=\infty
\end{array}
$$


where:

$$
\mu_{f}(s)=\int_{E_{s}} w(x) d x, \quad E_{s}=\left\{x \in \mathbb{R}^{n}:|f(x)|>s\right\}
$$

In general the Lorentz spaces are only quasinorms (they only have the triangle inequality up to a constant). However, here we've restricted $p$ and $q$ so that these spaces are normed. It's straightforward to check that $\|f\|_{p, p ; w}=\|f\|_{p ; w}$ and also that we get the usual non weighted spaces when $w(x) d x$ is Lebesgue measure. The function $\mu_{f}(s)$ is commonly referred to as the distribution function of $f$ with respect to the measure $w(x) d x$. Next we present some basic facts about Lorentz spaces.

Theorem 7.2 The following holds:

$$
\|f\|_{p, q_{2}, w} \leq\|f\|_{p, q_{1}, w} \quad \forall 1 \leq q_{1} \leq q_{2} \leq \infty
$$

\subsection{FOUNDATIONS OF THE METHOD}

The techniques applied in this chapter will be analogous to but significantly more abstract than those used in the $L_{p}$ case in previous chapters. In order to interpolate to obtain general convolution inequalities we will need preliminary inequalities to interpolate between as well as some new interpolation theorems applicable to Lorentz spaces. Through the work of Hunt [8] and Stein and Weiss [9] we will be able to obtain the necessary inequalities simply by getting a handle of the behavior of the convolution operator on characterstic functions of sets of finite measure. To begin we will use the following boundedness condition that need only hold on this relatively small class of functions. As presented by Stein and Weiss [9]:

Definition 7.3(Restricted Weak Type) An operator $T$ mapping $w(x) d x$ measurable functions into $\mu$ measurable functions is said to be of restricted weak type $(p, q)$ 
if there exists some constant $C$ independent of the sets $E$ such that:

$$
s \mu_{T} \chi_{E}(s)^{\frac{1}{q}} \leq C\left\|\chi_{E}\right\|_{p ; w} \quad \forall s>0
$$

where $E$ is of finite $w(x) d x$ measure.

Remark 7.4: In the case that $\mu$ is of the form $w^{\prime}(x) d x$ this condition is exactly equivalent to $\left\|T \chi_{E}\right\|_{q, \infty, w^{\prime}} \leq C\left\|\chi_{E}\right\|_{p, 1, w}$ where $C$ is independent of $E$.

Contrast this with the more general notion of weak type which is simply a more conventional boundedness condition on simple functions. Our analysis of the convolution operator will involve fixing one component at a time and analyzing each as a conventional mono-linear operator. The following theorem due to Stein and Weiss [9] will help us decide when that operator is of restricted weak type.

Theorem 7.5 An operator $T$ is of restricted weak type $(p, q)$ with constant $C_{1}$ if and only if there exists some constant $C$ such that for all sets $F$ and $G$ of finite Lebesgue measure:

$$
\int_{G} T \chi_{F}(x) w^{\prime}(x) d x \leq C\left(\int_{F} w(x) d x\right)^{\frac{1}{p}}\left(\int_{G} w^{\prime}(x) d x\right)^{\frac{1}{q^{\prime}}}
$$

where $C_{1} \approx C$.

Finally, the following theorem due to Hunt [8] will enable us to extend operators satisfying restricted weak type conditions to all measurable functions. This will give us the inequalities that we can interpolate between. As stated by Hunt:

Theorem 7.6 Suppose $T f$ is a $w^{\prime}(x) d x$ measureable function for each simple function 
$f \in L_{p, 1, w}$ and:

$$
\left\|T \chi_{E}\right\|_{q, \infty ; w^{\prime}} \leq C\left\|\chi_{E}\right\|_{p, 1, w}
$$

for every set $E$ with finite $w(x) d x$ measure. Then $T$ can be extended to a bounded operator on all of $L_{p, 1, w}$ :

$$
\|T f\|_{q, \infty ; w^{\prime}} \leq C\|f\|_{p, 1, w}
$$

Remark 7.7: Due to the first remark this simply states that restricted weak type operators can be extended to bounded and linear operators on the entire space of measurable functions.

Similarly to our previous analyses we seek sufficient conditions on indices $\alpha, \beta, \gamma, p, p_{0}, p_{1}, q, q_{1}, q_{2}$ so that:

$$
\|f * g\|_{p, q, w} \leq C\|f\|_{p_{0}, q_{0}, w_{0}}\|g\|_{p_{1}, q_{1}, w_{1}}
$$

This notation now represents the following as discussed below:

$$
w=(1+|x|)^{\gamma p}, w_{0}=(1+|x|)^{\alpha p_{0}}, w_{1}=(1+|x|)^{\beta p_{1}}
$$

and of course the constant $C$ is independent of the functions $f$ and $g$. For the rest of this chapter assume that $w, w_{0}$, and $w_{1}$ represent the weights defined above. In this chapter we will stray from our usual notation for norms to make applications of certain theorems clearer. In previous chapters we've been using the notation $\|u\|_{p, w}$ to represent $L^{p}$ spaces weighted by $w(x)$ and $\|u\|_{p, \alpha}$ when the weights are of the form $w(x)=(1+|x|)^{\alpha p}$. Take note of the fact that in this section we will continue to write $\|f\|_{p, q, w}$ even when the weights are of the form $w(x)=(1+|x|)^{\alpha p}$ in order to make certain applications of theorems more manageable. Finally, we present the 
interpolation theorems to be used in this analysis. The first one is reffered to as the Off-Diagonal Marcinkiewicz Interpolation Theorem in [10]. See Theorem 1.4.19

Theorem 7.8 Suppose that $T$ is an operator satisfying:

$$
\|T f\|_{p_{i}^{\prime}, \infty ; w^{\prime}} \leq C_{i}\|f\|_{p_{i}, 1, w} \quad i=0,1
$$

Then T also satisfies:

$$
\|T f\|_{p_{t}^{\prime}, r ; w^{\prime}} \leq C\|f\|_{p_{t}, r, w} \quad \forall 1 \leq r \leq \infty \quad \forall 0<t<1
$$

with $C \approx \max \left\{C_{0}, C_{1}\right\}$, provided that:

$$
\begin{aligned}
& \frac{1}{p_{t}}=\frac{1-t}{p_{0}}+\frac{t}{p_{1}} \\
& \frac{1}{p_{t}^{\prime}}=\frac{1-t}{p_{0}^{\prime}}+\frac{t}{p_{1}^{\prime}}
\end{aligned}
$$

Notice that in Theorem 7.8, $t$ parameterizes a line between $\left(\frac{1}{p_{0}}, \frac{1}{p_{0}^{\prime}}\right)$ and $\left(\frac{1}{p_{1}}, \frac{1}{p_{1}^{\prime}}\right)$. Therefore the previous two equations are equivalent to requiring that $\left(\frac{1}{p_{t}}, \frac{1}{p_{t}^{\prime}}\right)$ lies on the open line segment connecting the points $\left(\frac{1}{p_{0}}, \frac{1}{p_{0}^{\prime}}\right)$ and $\left(\frac{1}{p_{1}}, \frac{1}{p_{1}^{\prime}}\right)$ in the $\left(\frac{1}{p_{t}}, \frac{1}{p_{t}^{\prime}}\right)$ plane. Understanding Theorem 7.8 in a geometric sense will be crucial to understanding proofs that follow. We will be applying Theorem 7.8 by constructing lines as opposed to finding valid values for $t$. Visually the resulting inequality holds for any point on the following line segment:

Theorem 7.9 Suppose that $T$ is a multilinear operator satisfying:

$$
\|T(f, g)\|_{p_{i}^{\prime \prime}, q_{i}^{\prime \prime}, w^{\prime}} \leq C_{i}\|f\|_{p_{i}^{\prime}, q_{i}^{\prime}, w^{\prime}}\|g\|_{p_{i}, q_{i}, w}, \quad i=0,1
$$




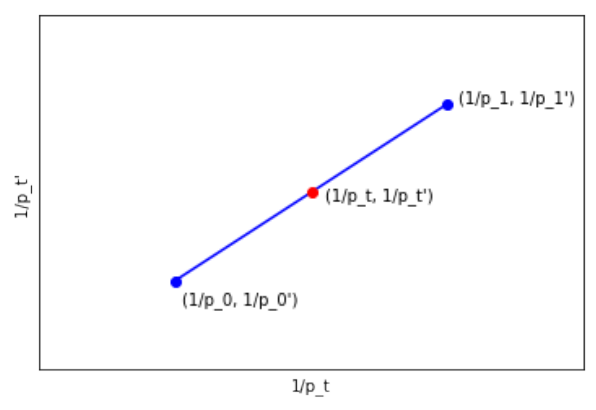

Then T also satisfies:

$$
\|T(f, g)\|_{p_{t}^{\prime \prime}, q_{t}^{\prime \prime}, w^{\prime}} \leq C\|f\|_{p_{t}^{\prime}, q_{t}^{\prime}, w^{\prime}}\|g\|_{p_{t}, q_{t}, w}, \quad 0<t<1
$$

with $C \approx C_{0}^{1-t} C_{1}^{t}$, whenever:

$$
\begin{aligned}
\frac{1}{p_{t}} & =\frac{1}{p_{0}}(1-t)+\frac{1}{p_{1}} t, \quad \frac{1}{p_{t}^{\prime}}=\frac{1}{p_{0}^{\prime}}(1-t)+\frac{1}{p_{1}^{\prime}} t, \quad \frac{1}{p_{t}^{\prime \prime}}=\frac{1}{p_{0}^{\prime \prime}}(1-t)+\frac{1}{p_{1}^{\prime \prime}} t \\
\frac{1}{q_{t}} & =\frac{1}{q_{0}}(1-t)+\frac{1}{q_{1}} t, \quad \frac{1}{q_{t}^{\prime}}=\frac{1}{q_{0}^{\prime}}(1-t)+\frac{1}{q_{1}^{\prime}} t, \quad \frac{1}{q_{t}^{\prime \prime}}=\frac{1}{q_{0}^{\prime \prime}}(1-t)+\frac{1}{q_{1}^{\prime \prime}} t
\end{aligned}
$$

Remark 7.10 It is possible to state Theorem 7.9 in the language of lines much like Theorem 7.8. The variable $t$ parameterizes two line segments in $\left(\frac{1}{p_{t}}, \frac{1}{p_{t}^{\prime}}, \frac{1}{p_{t}^{\prime \prime}}\right)$ and $\left(\frac{1}{q_{t}}, \frac{1}{q_{t}^{\prime}}, \frac{1}{q_{t}^{\prime \prime}}\right)$ space. But for our purposes here, the chosen language will be more useful. Finally, we have the main theorem of this chapter.

\subsection{THE MAIN LORENTZ SPACE RESULT}

Theorem 7.11 (Convolution on the Lorentz spaces) There exists some constant $C$ independent of the functions $f$ and $g$ such that:

$$
\|f * g\|_{p, q, w} \leq C\|f\|_{p_{0}, q_{0}, w_{0}}\|g\|_{p_{1}, q_{1}, w_{1}}
$$


Provided that $1<p, p_{0}, p_{1}<\infty, 0 \leq \frac{1}{q} \leq \frac{1}{q_{0}}+\frac{1}{q_{1}} \leq 1$, and one of the following sets of conditions holds:

$$
\begin{array}{r}
B_{1}: \\
0<1+\frac{1}{p}-\frac{1}{p_{0}}-\frac{1}{p_{1}}<1 \\
p_{0}<p \\
\alpha+\beta-\gamma \geq n+\frac{n}{p}-\frac{n}{p_{0}}-\frac{n}{p_{1}} \\
\alpha-\gamma, \beta-\gamma, \alpha+\beta>0 \\
\alpha-\gamma>n+\frac{n}{p}-\frac{n}{p_{0}}-\frac{n}{p_{1}} \\
\beta-\gamma>\frac{n}{p}-\frac{n}{p_{1}} \\
\alpha+\beta>n-\frac{n}{p_{0}}-\frac{n}{p_{1}}
\end{array}
$$

$B_{2}:$

$$
\begin{array}{r}
0<1+\frac{1}{p}-\frac{1}{p_{0}}-\frac{1}{p_{1}}<\frac{1}{p} \\
\alpha+\beta-\gamma \geq n+\frac{n}{p}-\frac{n}{p_{0}}-\frac{n}{p_{1}} \\
\alpha+\beta-2 \gamma>n+\frac{n}{p}-\frac{n}{p_{0}}-\frac{n}{p_{1}} \\
\alpha+\beta>0
\end{array}
$$$$
\alpha-\gamma, \beta-\gamma>0
$$$$
\beta-\gamma>\frac{n}{p}-\frac{n}{p_{1}}
$$$$
\alpha-\gamma>\frac{n}{p}-\frac{n}{p_{0}}
$$ 


$$
\begin{array}{r}
B_{3}: \\
\frac{1}{p_{0}}+\frac{1}{p_{1}}<1 \\
p<p_{0}, p<p_{1} \\
\alpha-\gamma>\frac{n}{p}-\frac{n}{p_{0}} \\
\beta-\gamma>\frac{n}{p}-\frac{n}{p_{1}} \\
\alpha+\beta-\frac{n}{p_{0}}-\frac{n}{p_{1}} \\
\alpha+\beta-\gamma>n+\frac{n}{p}-\frac{n}{p_{0}}-\frac{n}{p_{1}}
\end{array}
$$

$$
\begin{array}{r}
B_{4}: \\
0<1+\frac{1}{p}-\frac{1}{p_{0}}-\frac{1}{p_{1}}<1 \\
p_{0} \leq p, \quad p_{1} \leq p \\
\alpha+\beta-\gamma>n+\frac{n}{p}-\frac{n}{p_{0}}-\frac{n}{p_{1}} \\
\alpha-\gamma, \beta-\gamma, \alpha+\beta>0 \\
2 \beta+\alpha-\gamma>n+\frac{n}{p}-\frac{n}{p_{0}}-\frac{n}{p_{1}} \\
2 \alpha+\beta-\gamma>n+\frac{n}{p}-\frac{n}{p_{0}}-\frac{n}{p_{1}} \\
\alpha+\beta>n-\frac{n}{p_{0}}-\frac{n}{p_{1}}
\end{array}
$$

The proof will make direct use of the previously obtained weighted $L^{p}$ space convolution inequalities. We now present several important theorems that will be used.

Theorem 7.12 If $B_{3}$ is satisfied then there exists some constant $C$ such that for 
all $F, G, H \subset \mathbb{R}^{n}$ of finite Lebesgue measure we have:

$$
\begin{gathered}
\int_{H} \chi_{F} * \chi_{G}(x)(1+|x|)^{\gamma p} d x \\
\leq C\left(\int_{F}(1+|x|)^{\alpha p_{0}} d x\right)^{\frac{1}{p_{0}}}\left(\int_{G}(1+|x|)^{\beta p_{1}} d x\right)^{\frac{1}{p_{1}}}\left(\int_{H}(1+|x|)^{\gamma p} d x\right)^{\frac{1}{p^{\prime}}}
\end{gathered}
$$

where $p^{\prime}$ denotes the Holder conjugate of $p$.

(Proof of Theorem 7.12) We begin by considering the following three sets:

$$
\begin{gathered}
E_{1}=\{|y|>2|x|\} \\
E_{2}=\left\{|y|<2|x|,|x-y|>\frac{|x|}{2}\right\} \\
E_{3}=\left\{|y|<2|x|,|x-y| \leq \frac{|x|}{2}\right\}
\end{gathered}
$$

And then define:

$$
I_{i}(x)=\int_{E_{i}} \chi_{F}(y) \chi_{G}(x-y) d y \quad i=1,2,3
$$

so that:

$$
\chi_{F} * \chi_{G}(x)=I_{1}(x)+I_{2}(x)+I_{3}(x)
$$

Since $(1+|x-y|) \approx(1+|y|)$ on $E_{1}$ we have:

$$
I_{1}(x) \leq C \int_{E_{1}} \chi_{F}(y)(1+|y|)^{\alpha} \chi_{G}(x-y)(1+|x-y|)^{\beta}(1+|y|)^{-\alpha-\beta} d y
$$

With indices $p_{0}, p_{1}$, and $s$ with $\frac{1}{s}=1-\frac{1}{p_{0}}-\frac{1}{p_{1}}$ we apply Holder's inequality to the functions $\chi_{F}(y)(1+|y|)^{\alpha}, \chi_{G}(x-y)(1+|x-y|)^{\beta}$, and $\chi_{E_{1}}(1+|y|)^{-\alpha-\beta}$ to get: 


$$
I_{1}(x) \leq C\left(\int_{F}(1+|x|)^{\alpha p_{0}} d x\right)^{\frac{1}{p_{0}}}\left(\int_{G}(1+|x|)^{\beta p_{1}} d x\right)^{\frac{1}{p_{1}}}\left(\int_{E_{1}}(1+|y|)^{-(\alpha+\beta) s} d y\right)^{\frac{1}{s}}
$$

Since $1<\frac{1}{p_{0}}+\frac{\alpha}{n}+\frac{1}{p_{1}}+\frac{\beta}{n} \Longrightarrow(\alpha+\beta) s>n$ we have that:

$$
\left(\int_{E_{1}}(1+|y|)^{-(\alpha+\beta) s} d y\right)^{\frac{1}{s}} \leq C(1+|x|)^{\frac{n}{s}-(\alpha+\beta)}
$$

so that:

$$
\begin{gathered}
\int_{H} I_{1}(x)(1+|x|)^{\gamma p} d x \\
\leq C\left(\int_{F}(1+|x|)^{\alpha p_{0}} d x\right)^{\frac{1}{p_{0}}}\left(\int_{G}(1+|x|)^{\beta p_{1}} d x\right)^{\frac{1}{p_{1}}} \int_{H}(1+|x|)^{\frac{n}{s}-(\alpha+\beta)}(1+|x|)^{\gamma p} d x
\end{gathered}
$$

Using $(1+|x|)^{\gamma p} d x$ as the measure we may apply Holder's inequality with indices $p$ and $p^{\prime}$ so that:

$$
\begin{gathered}
\int_{H}(1+|x|)^{\frac{n}{s}-(\alpha+\beta)}(1+|x|)^{\gamma p} d x \\
\leq\left(\int(1+|x|)^{\frac{n p}{s}-(\alpha+\beta) p+\gamma p} d x\right)^{\frac{1}{p}}\left(\int_{H}(1+|x|)^{\gamma p} d x\right)^{\frac{1}{p^{\prime}}}
\end{gathered}
$$

The lefthand factor here is a constant since $1+\frac{1}{p}+\frac{\gamma}{n}<\frac{1}{p_{0}}+\frac{\alpha}{n}+\frac{1}{p_{1}}+\frac{\beta}{n} \Longrightarrow$ $(\alpha+\beta) p-\gamma p-\frac{n p}{s}>n$. Therefore we've obtained:

$$
\begin{gathered}
\int_{H} I_{1}(x)(1+|x|)^{\gamma p} d x \\
\leq C\left(\int_{F}(1+|x|)^{\alpha p_{0}} d x\right)^{\frac{1}{p_{0}}}\left(\int_{G}(1+|x|)^{\beta p_{1}} d x\right)^{\frac{1}{p_{1}}}\left(\int_{H}(1+|x|)^{\gamma p} d x\right)^{\frac{1}{p^{\prime}}}
\end{gathered}
$$


On $E_{2}$ we have $(1+|x-y|) \approx(1+|x|)$ so that:

$$
\begin{gathered}
\int_{H} I_{2}(x)(1+|x|)^{\gamma p} d x \\
\leq C \int_{H} \int_{E_{2}} \chi_{F}(y) \chi_{G}(x-y)(1+|x-y|)^{\beta}(1+|x|)^{\gamma p-\gamma}(1+|x|)^{\gamma-\beta} d y d x
\end{gathered}
$$

A reversal of the order of integration gives:

$$
\begin{gathered}
\int_{H} I_{2}(x)(1+|x|)^{\gamma p} d x \\
\leq C \int_{F} \int \chi_{G}(x-y)(1+|x-y|)^{\beta} \chi_{H}(x)(1+|x|)^{\gamma p-\gamma} \chi_{E_{2}}(x)(1+|x|)^{\gamma-\beta} d x d y
\end{gathered}
$$

We apply Holder's inequality with indices $p_{1}, \frac{p}{p-1}=p^{\prime}$, and $s$ with $\frac{1}{s}=\frac{1}{p}-\frac{1}{p_{1}}$ to the functions $\chi_{G}(x-y)(1+|x-y|)^{\beta}, \chi_{H}(x)(1+|x|)^{\gamma p-\gamma}$, and $\chi_{E_{2}}(x)(1+|x|)^{\gamma-\beta}$ to get:

$$
\begin{gathered}
\int_{H} I_{2}(x)(1+|x|)^{\gamma p} d x \\
\leq C\left(\int_{G}(1+|x|)^{\beta p_{1}} d x\right)^{\frac{1}{p_{1}}}\left(\int_{H}(1+|x|)^{\gamma p} d x\right)^{\frac{1}{p^{\prime}}} \int_{F}\left(\int_{E_{2}}(1+|x|)^{(\gamma-\beta) s} d x\right)^{\frac{1}{s}} d y
\end{gathered}
$$

Using the fact that $\frac{1}{p}+\frac{\gamma}{n}<\frac{1}{p_{1}}+\frac{\beta}{n} \Longrightarrow(\beta-\gamma) s>n$, applying Holder's inequality, and then using the fact that $1+\frac{1}{p}+\frac{\gamma}{n}<\frac{1}{p_{0}}+\frac{\alpha}{n}+\frac{1}{p_{1}}+\frac{\beta}{n} \Longrightarrow\left(\alpha+\beta-\gamma-\frac{n}{s}\right) p_{0}^{\prime}>n$ 
we get:

$$
\begin{array}{r}
\int_{F}\left(\int_{E_{2}}(1+|x|)^{(\gamma-\beta) s} d x\right)^{\frac{1}{s}} d y \\
\leq \int_{F}\left(\int_{\left\{|x|>\frac{|y|}{2}\right\}}(1+|x|)^{(\gamma-\beta) s} d x\right)^{\frac{1}{s}} d y \\
\leq C \int_{F}(1+|y|)^{\frac{n}{s}+\gamma-\beta} d y \\
=C \int_{F}(1+|x|)^{\frac{n}{s}+\gamma-\beta-\alpha+\alpha} d x \\
\leq C\left(\int(1+|x|)^{\left(\frac{n}{s}+\gamma-\beta-\alpha\right) p_{0}^{\prime}} d x\right)^{\frac{1}{p_{0}^{\prime}}}\left(\int_{F}(1+|x|)^{\alpha p_{0}} d x\right)^{\frac{1}{p_{0}}} \\
=C\left(\int_{F}(1+|x|)^{\alpha p_{0}} d x\right)^{\frac{1}{p_{0}}}
\end{array}
$$

To handle the final case, we change variables, then reverse the order of integration to get:

$$
\begin{aligned}
\int_{H} I_{3}(x)(1+|x|)^{\gamma p} d x & =\int_{H} \int \chi_{F}(x-z) \chi_{G}(z) \chi_{E_{3}}(x-z)(1+|x|)^{\gamma p} d z d x \\
& =\int_{G} \int \chi_{F}(x-z) \chi_{H}(x) \chi_{E_{3}}(x-z)(1+|x|)^{\gamma p} d x d z
\end{aligned}
$$

with $E_{3}$ expressed as $\left\{|x-z|<2|x|,|z| \leq \frac{|x|}{2}\right\}$. On $E_{3}$ we have $(1+|x-z|) \approx(1+|x|)$ so that:

$$
\begin{gathered}
\int_{H} I_{3}(x)(1+|x|)^{\gamma p} d x \\
\leq C \int_{G} \int \chi_{F}(x-z)(1+|x-z|)^{\alpha} \chi_{H}(x)(1+|x|)^{\gamma p-\gamma} \chi_{E_{3}}(x-z)(1+|x-z|)^{\gamma-\alpha} d x d z
\end{gathered}
$$

Similar to the $E_{2}$ case we apply Holder's inequality with indices $p_{0}, p^{\prime}$ and $s$ with $\frac{1}{s}=\frac{1}{p}-\frac{1}{p_{0}}$ to get:

$$
\begin{gathered}
\int_{H} I_{3}(x)(1+|x|)^{\gamma p} d x \leq \\
C\left(\int_{F}(1+|x|)^{\alpha p_{0}} d x\right)^{\frac{1}{p_{0}}}\left(\int_{H}(1+|x|)^{\gamma p} d x\right)^{\frac{1}{p^{\prime}}}\left(\int_{G}\left(\int_{\{|x| \geq 2|z|\}}(1+|x|)^{(\gamma-\alpha) s} d x\right)^{\frac{1}{s}} d z\right)
\end{gathered}
$$


where the integral over G is handled using $\frac{1}{p}+\frac{\gamma}{n}<\frac{1}{p_{0}}+\frac{\alpha}{n} \Longrightarrow(\alpha-\gamma) s>n$, Holder's inequality, and $1+\frac{1}{p}+\frac{\gamma}{n}<\frac{1}{p_{0}}+\frac{\alpha}{n}+\frac{1}{p_{1}}+\frac{\beta}{n} \Longrightarrow\left(\alpha+\beta-\gamma-\frac{n}{s}\right) p_{1}^{\prime}>n$.

(Proof of Theorem 7.11.) When $B_{3}$ is satisfied, we get (42) by Theorem 7.12. When $B_{1}, B_{2}$, or $B_{4}$ is satisfied, we apply Holder's inequality to the functions $\chi_{H}$ and $\chi_{F} * \chi_{G}$ and use the main $L^{p}$ space convolution inequality derived in Chapter 3:

$$
\begin{array}{r}
\int_{H} \chi_{F} * \chi_{G}(x)(1+|x|)^{\gamma p} d x \leq\left\|\chi_{F} * \chi_{G}\right\|_{p, w}\left\|\chi_{H}\right\|_{p^{\prime}, w} \\
\leq C\left\|\chi_{F}\right\|_{p_{0}, w_{0}}\left\|\chi_{G}\right\|_{p_{1}, w_{1}}\left\|\chi_{H}\right\|_{p^{\prime}, w}
\end{array}
$$

For a fixed $\mathrm{F}$, the operator $T_{F}\left(\chi_{G}\right)=\chi_{F} * \chi_{G}$ satisfies the integral inequality in Theorem 7.5 with constant essentially equal to $\left\|\chi_{F}\right\|_{p_{0}, w_{0}}$. Therefore $T_{F}$ is of restricted weak type $\left(p_{1}, p\right)$, meaning that for all sets $G$ with finite $w_{1}(x) d x$ measure we have:

$$
s\left(\int_{E_{s}} w(x) d x\right)^{\frac{1}{p}} \leq C\left\|\chi_{F}\right\|_{p_{0} ; w_{0}}\left\|\chi_{G}\right\|_{p_{1} ; w_{1}} \forall s>0
$$

where $E_{s}=\left\{x \in \mathbb{R}^{n}:\left|T_{F}\left(\chi_{G}\right)\right|>s\right\}$. A straightforward calculation shows that $\left\|\chi_{G}\right\|_{p_{1}, w}=\left\|\chi_{G}\right\|_{p_{1}, 1, w}$. Taking the supremum over all $s>0$ and extending $T_{F}$ using Theorem 7.6 gives:

$$
\left\|\chi_{F} * g\right\|_{p, \infty ; w} \leq C\left\|\chi_{F}\right\|_{p_{0}, 1 ; w_{0}}\|g\|_{p_{1}, 1 ; w_{1}}
$$

Considering the operator defined by $T_{g}\left(\chi_{F}\right)=\chi_{F} * g$ and applying Theorem 7.6 again we obtain:

$$
\|f * g\|_{p, \infty ; w} \leq C\|f\|_{p_{0}, 1 ; w_{0}}\|g\|_{p_{1}, 1 ; w_{1}}
$$

We now discuss separate cases in order to obtain the following inequality:

$$
\|f * g\|_{p, r ; w} \leq C\|f\|_{p_{0}, \infty ; w_{0}}\|g\|_{p_{1}, r ; w_{1}} \quad \forall 1 \leq r \leq \infty
$$


Equivalently, due to symmetry of the indices in the hypotheses,

$$
\|f * g\|_{p, r ; w} \leq C\|f\|_{p_{0}, r ; w_{0}}\|g\|_{p_{1}, \infty ; w_{1}} \quad \forall 1 \leq r \leq \infty
$$

Case 1: Suppose $B_{1}, B_{2}$, or $B_{3}$. In the first two cases there exists $K \geq 0$ so that $\alpha+\beta-\gamma=n+\frac{n}{p}-\frac{n}{p_{0}}-\frac{n}{p_{1}}+K$. In case $B_{3}$ we have that $K>0$. It's straightforward to check that $\left(\frac{1}{p_{0}}, \frac{1}{p}\right)$ lies on the line:

$$
1+\frac{1}{p_{t}^{\prime}}\left(1+\frac{c}{n}\right)=\frac{1}{p_{t}}\left(1+\frac{k}{n}\right)+\frac{1}{p_{1}}+\frac{\beta}{n}+K
$$

in the $\left(\frac{1}{p_{t}}, \frac{1}{p_{t}^{\prime}}\right)$ plane with $c=\gamma p$ and $k=\alpha p_{0}$. On this line it is possible to choose points $\left(\frac{1}{p_{0}^{\prime}}, \frac{1}{p^{\prime}}\right)$ and $\left(\frac{1}{p_{0}^{\prime \prime}}, \frac{1}{p^{\prime \prime}}\right)$ sufficiently close to $\left(\frac{1}{p_{0}}, \frac{1}{p}\right)$ with $\frac{1}{p_{0}^{\prime}}<\frac{1}{p_{0}}<\frac{1}{p_{0}^{\prime \prime}}$ and corresponding indices $\gamma^{\prime}, \gamma^{\prime \prime}, \alpha^{\prime}, \alpha^{\prime \prime}$ so that $\gamma p=\gamma^{\prime} p^{\prime}=\gamma^{\prime \prime} p^{\prime \prime}$ and $\alpha p_{0}=\alpha^{\prime} p_{0}^{\prime}=\alpha^{\prime \prime} p_{0}^{\prime \prime}$ so that the hypotheses of Theorem 7.11 remain true after replacement of the unprimed variables with the primed ones. We obtain the following two inequalities:

$$
\begin{aligned}
& \|f * g\|_{p^{\prime}, \infty ; w} \leq C\|f\|_{p_{0}^{\prime}, 1 ; w_{0}}\|g\|_{p_{1}, 1 ; w_{1}} \\
& \|f * g\|_{p^{\prime \prime}, \infty ; w} \leq C\|f\|_{p_{0}^{\prime \prime}, 1 ; w_{0}}\|g\|_{p_{1}, 1 ; w_{1}}
\end{aligned}
$$

By considering the operator defined by $T_{g}(f)=f * g$ we may use Theorem 7.8 to interpolate between these two inequalities to obtain:

$$
\|f * g\|_{p, r ; w} \leq C\|f\|_{p_{0}, r ; w_{0}}\|g\|_{p_{1}, 1 ; w_{1}} \quad \forall 1 \leq r \leq \infty
$$

We then obtain (43) by taking $r=\infty$ in (45) and similarly interpolating between two inequalities obtained by considering the operator $T_{f}(g)=f * g$ and the point $\left(\frac{1}{p_{1}}, \frac{1}{p}\right)$ on the line: 


$$
1+\frac{1}{p_{t}^{\prime}}\left(1+\frac{c}{n}\right)=\frac{1}{p_{0}}+\frac{\alpha}{n}+\frac{1}{p_{t}}\left(1+\frac{k}{n}\right)+K
$$

with $c=\gamma p$ and $k=\beta p_{1}$.

Case 2: Under the hypotheses of $B_{4}$ we select $K_{0}, K_{1} \geq 0$ so that $\frac{1}{p}=\frac{1}{p_{0}}+K_{0}$ and $\frac{1}{p}=\frac{1}{p_{1}}+K_{1}$. We proceed similarly to the previous two cases but instead with the following two lines:

$$
\begin{aligned}
& \frac{1}{p_{t}^{\prime}}=\frac{1}{p_{t}}+K_{0} \\
& \frac{1}{p_{t}^{\prime}}=\frac{1}{p_{t}}+K_{1}
\end{aligned}
$$

Note that again $\left(\frac{1}{p_{0}}, \frac{1}{p}\right)$ and $\left(\frac{1}{p_{1}}, \frac{1}{p}\right)$ lie on these lines but for certain boundary cases (in particular when either $K_{0}=0$ or $K_{1}=0$ ) we might lose $p_{0} \leq p$ or $p_{1} \leq p$ for points near $\left(\frac{1}{p_{0}}, \frac{1}{p}\right)$ and $\left(\frac{1}{p_{1}}, \frac{1}{p}\right)$. However this is okay since at least one of them will always be true. This will preserve our ability to use Holder's inequality and the convolution inequality derived in Chapter 3 for the close points as was done at the beginning of this proof. Thus we have established (43) and (44) for all cases in the hypotheses. Take $r=1$ in (43) and (44). For any choice of $1<r<\infty$ select $t=\frac{1}{r}$ and interpolate using Theorem 7.9 to obtain:

$$
\|f * g\|_{p, 1 ; w} \leq C\|f\|_{p_{0}, r ; w_{0}}\|g\|_{p_{1}, r^{\prime} ; w_{1}} \quad \forall 1 \leq r \leq \infty
$$

where $r^{\prime}$ denotes the Holder conjugate of $r$. The cases where $r=1$ and $r=\infty$ in (46) are given to us by (44). Now take $r=q_{0}$ in (44) and (46) and interpolate by applying the same theorem with $t=\frac{q_{0}}{q_{1}\left(q_{0}-1\right)}$ to get:

$$
\|f * g\|_{p, q ; w} \leq C\|f\|_{p_{0}, q_{0} ; w_{0}}\|g\|_{p_{1}, q_{1} ; w_{1}}
$$


whenever $\frac{1}{q}=\frac{1}{q_{0}}+\frac{1}{q_{1}}$. We then get (41) from Theorem 7.2. 


\section{CHAPTER 8 \\ OPPORTUNITIES FOR FURTHER RESEARCH}

The work done in this dissertation provides many opportunities for further research. The purpose of developing convolution inequalities in weighted Lorentz spaces was to pave the way for obtaining solutions to partial differential equations with initial data in weighted Lorentz spaces. Achieving this result will require sophisticated estimates in Lorentz spaces analogous to those made in chapter 6 with weighted $L_{p}$ spaces. In addition the possible inclusion of Gevrey norms in Lorentz spaces analogous to those used in chapter 6 will require making some decisions on where to include the exponential factor in the Lorentz norms.

In addition further research could entail investigating weights more general than $(1+|x|)^{\alpha p}$ enabling the techniques presented in this dissertation to be applied to far more use cases. 


\section{REFERENCES}

[1] Hardy G. H., Littlewood J.E., Some properties of fractional integrals, I; Math.Zeit.,27(1928) pp. $565-606$

[2]Stein E.M., Weiss G., Fractional Integrals on n-dimensional Euclidean Space Journal of Mathematics and Mechanics, Vol. 7, No. 4 (July, 1958), pp. 503-514

[3] Swanson D., (2018) A Simplified Proof of the Stein-Weiss Inequality and a Characterization of a Weighted Inequality

[4] Ziemer W. (1989), Weakly Differentiable Functions, Springer, New York NY

[5] Bergh J., Lofstrom J. (1976), Interpolation Spaces Springer-Verlag, Berlin

[6] Kristansson K., (2002) Decreasing Rearrangement and Lorentz L(p,q) spaces

[7] Kerman R. A., Convolution Theorems with Weights Transactions of the American Mathematical Society, Vol. 280, Number 1, November 1983

[8] R. A. Hunt, On L(p,q) spaces, Enseign. Math. (2) 12 (1966), 249-276.

[9] Stein E.M., Weiss G., Interpolation of Operators with Change of Measures Transactions of the American Mathematical Society, Vol. 87, No. 1 (Jan., 1958), pp. $159-172$

[10] Grafakos L., (2008) Classical Fourier Analysis, 2e, Springer, New York, NY

[11] Calderon A. P., Intermediate Spaces and Interpolation, the Complex Method Studia Mathematica. T. XXIV. (1964)

[12] Biswas A., Swanson D., Existence and generalized Gevrey reguliarty of solutions to the Kuramoto-Sivashinsky equation in $\mathbb{R}^{n}$ J. Differential Equations 240 (2007) 145163

[13] Sell G., You Y., (2002), Dynamics of Evolutionary Equations Springer-Verlag, New York NY 


\section{CURRICULUM VITAE \\ Matthew Reynolds}

\section{Academic Record}

University of Louisville, Louisville, $K Y$

Ph.D. in Applied and Industrial Mathematics

Advisor: David Swanson

Expected August 2020

University of Louisville, Louisville, $K Y$

M. A. Mathematics

May 2016

University of Louisville, Louisville, $K Y$

B. S. Mathematics

May 2014

\section{Teaching Experience}

University of Louisville Graduate Teaching Assistant 2015-2020

Lead Instructor

- MA206: Calculus II Fall 2020

- MA205: Calculus I Spring 2019

- MA111: College Algebra 2014-2018 